ARTICLE

\title{
Locus specific epigenetic modalities of random allelic expression imbalance
}

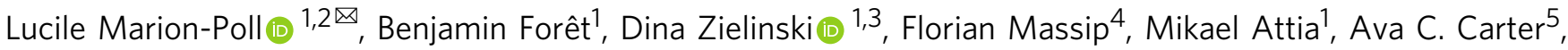
Laurène Syx ${ }^{1,3}$, Howard Y. Chang (iD ${ }^{5}$, Anne-Valerie Gendrel (iD ${ }^{1 凶} \&$ Edith Heard ${ }^{1,2,6 凶}$

Most autosomal genes are thought to be expressed from both alleles, with some notable exceptions, including imprinted genes and genes showing random monoallelic expression (RME). The extent and nature of RME has been the subject of debate. Here we investigate the expression of several candidate RME genes in F1 hybrid mouse cells before and after differentiation, to define how they become persistently, monoallelically expressed. Clonal monoallelic expression is not present in embryonic stem cells, but we observe high frequencies of monoallelism in neuronal progenitor cells by assessing expression status in more than 200 clones. We uncover unforeseen modes of allelic expression that appear to be genespecific and epigenetically regulated. This non-canonical allelic regulation has important implications for development and disease, including autosomal dominant disorders and opens up therapeutic perspectives.

\footnotetext{
${ }^{1}$ Institut Curie, PSL Research University, CNRS UMR3215, INSERM U934, Paris, France. ${ }^{2}$ Directors' research, European Molecular Biology Laboratory (EMBL), Heidelberg, Germany. ${ }^{3}$ Institut Curie, PSL Research University, INSERM U900, Mines ParisTech, Paris, France. ${ }^{4}$ Berlin Institute for Medical Systems Biology, Max Delbrück Center for Molecular Medicine, Berlin, Germany. ${ }^{5}$ Center for Personal Dynamic Regulomes, Stanford University, Stanford, CA, USA

${ }^{6}$ Collège de France, Paris, France. ${ }^{凶}$ email: lucile.marion-poll@embl.de; anne.gendrel@medicina.ulisboa.pt; edith.heard@embl.org
} 
G enes are generally thought to be expressed from both alleles, even if their expression is not necessarily equal nor synchronised. There are some exceptions where gene expression occurs exclusively from one allele. This can be due to heterozygous loss-of-function mutations, allelic exclusion via DNA rearrangements (e.g. Immunoglobulins ${ }^{1}$ ) or epigenetically based differences leading to clonally inherited monoallelic expression. Epigenetically based monoallelic expression is found at parentally imprinted $\mathrm{loci}^{2}$, on one $\mathrm{X}$ chromosome in females (X-chromosome inactivation) ${ }^{3}$ and for olfactory receptor genes ${ }^{4}$. A less well-understood category concerns autosomal, clonally heritable 'random monoallelically expressed' (RME) genes (estimated to comprise 1 to $10 \%$ of genes) ${ }^{5}$. RME genes were identified through numerous genome-wide allele-specific analyses of gene expression in single-cell derived clones from hybrid cell lines ${ }^{6-14}$. These studies showed that some genes can be expressed monoallelically from the maternal or paternal allele, or biallelically, and that these expression patterns are inherited during cell division. These RME genes ${ }^{15}$ belong to a wide range of gene ontologies and are enriched in cell surface receptors, developmental regulators, and are often associated with human autosomal dominant diseases ${ }^{6,10,11}$.

RME has potentially important implications in development and disease. It might confer many advantages to the organism, such as generating cellular diversity, enhancing adaptability or regulating gene dosage, but this remains to be investigated ${ }^{16}$. On the other hand, RME may also be detrimental, particularly in the context of heterozygous mutations, as a proportion of cells could express the mutated allele only, thereby contributing to pathological phenotypes in a non-classical manner. Indeed some autosomal dominant disorders involving putative RME loci may be a result of functional nullisomy in a proportion of cells rather than haploinsufficiency ${ }^{5}$.

However, the extent to which clonal and stable RME occurs is still somewhat debated ${ }^{17,18}$. First, stable RME is often confounded with transient RME observed in single cells, which originates from transcriptional bursting. Although there have been some efforts to identify RME in vivo ${ }^{11,19}$, transient and stable RME cannot be readily distinguished at the single-cell level (by RNAseq or nascent RNA-FISH) without information about clonality. Second, RME genes are often associated with low expression, raising several issues. A poor detection of lowly expressed genes could lead to the unreliability of the allelic measurements. Also, monoallelic expression of a virtually silent gene could arise through incomplete silencing of one allele and subsequent clonal propagation, which would not be functionally relevant. There are therefore critical parameters to consider, and careful analytical and statistical methods are necessary to confidently identify stable RME, particularly in vivo. In addition, the mechanisms underlying stable RME are largely unexplored. In previous studies ${ }^{6-14}$, the number of single-cell derived clones analysed in parallel was rather limited (4-16), rendering any assumptions on the extent of RME and on the mechanisms involved difficult ${ }^{20}$.

Here we assess whether and how some autosomal genes that we previously identified as being RME are prone to persistent and stable monoallelic expression in clones of neural progenitor cells (NPC). We also explore the mechanisms underlying RME and its consequences on gene dosage.

The frequencies of monoallelic expression for a subset of genes involved in disease were assessed in more than 200 newly derived NPC clones. Unexpectedly, we found that genes previously identified as RME show very specific modalities of allelic expression. We propose that these loci are subject to random allelic expression imbalance (RAExI), with one or several stable states of allelic expression per gene. RAExI consists of a range of different expression modalities, and includes RME as one subtype of RAExI. We also evaluate whether these modalities are determined prior to differentiation. Importantly we assess the consequences of allele-specific expression on gene dosage and on cellular diversity. Finally, we investigate the epigenetic mechanisms involved in the maintenance of differential allelic expression. Taken together, our results reveal unforeseen modes of allelic expression, that appear to be gene-specific and epigenetically regulated. This noncanonical allelic regulation affects the genes of this study, which are involved in development and associated with diseases, but the scope of RAExI could be much broader, with major physiological and pathological implications.

\section{Results}

The study of RME reveals the existence of RAExI. In order to investigate the extent and nature of RME in depth, we selected 12 genes that were previously found to be RME in NPCs $(A 2 m$, Acyp2, Bag3, Cnrip1, Eya1, Eya2, Eya4, Grik2, Kcnq2, Ptk2b and Snca $)^{11}$ or in B-lymphoblastoid clones $(A p p)^{6}$. We also investigated a previously described biallelically expressed gene (Eya3). Characteristics of these genes and their association with diseases are summarised in Table 1.

In order to assess the frequency with which these genes show RME, we differentiated male or female hybrid (129/Sv x Castaneus) mouse ES cells (ESCs) into NPCs, as previously described $^{11,21}$ (Fig. 1 and Supplementary Fig. 1). NPCs have the advantage of self-renewing continuously and are clonogenic, as well as being able to differentiate further into neurons,

Table 1 Genes of interest, selected from previous genome-wide studies.

\begin{tabular}{|c|c|c|}
\hline Gene & Name & Disease association \\
\hline $\mathrm{A} 2 \mathrm{~m}$ & Alpha-2-macroglobulin & Alzheimer's disease, alpha-2-macroglobulin deficiency \\
\hline Acyp2 & Acylphosphatase 2 & Cancer \\
\hline App & Amyloid beta precursor protein & Alzheimer's disease (dominant) \\
\hline Bag3 & $\mathrm{BCL} 2$-binding athanogene 3 & Myopathy, cardiomyopathy, neuropathy (dominant) \\
\hline Cnrip1 & $\begin{array}{l}\text { Cannabinoid receptor- } \\
\text { interacting protein } 1\end{array}$ & \\
\hline Eya1 & Eyes absent homologue 1 & Branchio-otic \& branchio-oto-renal syndromes (dominant) \\
\hline Eya2 & Eyes absent homologue 2 & \\
\hline Eya3 & Eyes absent homologue 3 & (biallelic control) \\
\hline Eya4 & Eyes absent homologue 4 & Cardiomyopathy, deafness (dominant) \\
\hline Grik2 & Glutamate receptor GluR6 & Mental retardation, epilepsy (recessive) \\
\hline Kenq2 & Potassium channel Kv7.2 & Epilepsy, myokymia (dominant) \\
\hline Ptk2b & Protein-tyrosine kinase 2 Beta & Osteoporosis, Alzheimer's disease \\
\hline Snca & Alpha-synuclein & Parkinson's disease (dominant) \\
\hline
\end{tabular}



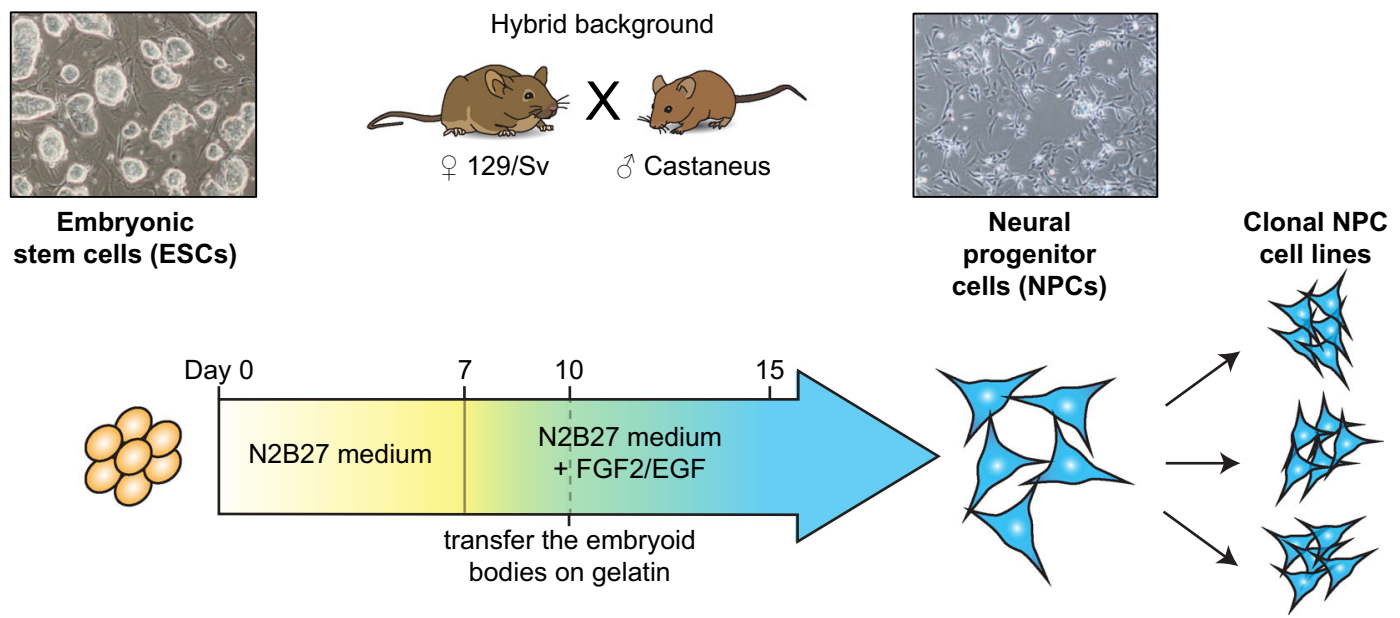

2 cell lines:

$\mathrm{F} 121.6(X X)$

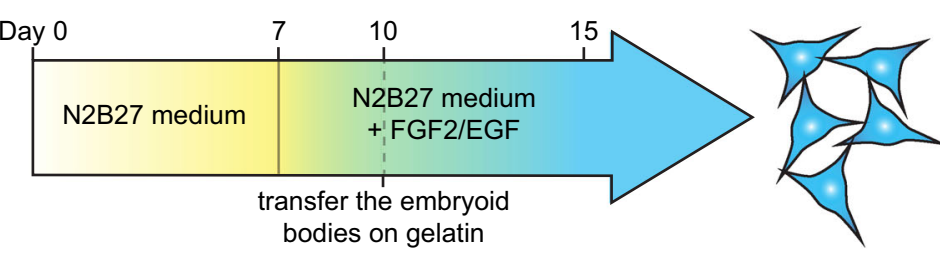

22-61 clones /experiment 249 clones total

Fig. 1 Experimental strategy to assess the frequency of monoallelic expression. Generation of NPC clonal cell lines (249 clones in total) from two hybrid ESC lines after six independent in vitro differentiation experiments.

oligodendrocytes or astrocytes in vitro ${ }^{21}$. We established 249 independent NPC clonal cell lines from single cells, from six independent differentiation experiments (Supplementary Table 1). The analysis of such a large number of clones confers better statistical power and robustness compared to all previous studies $^{6-14}$.

To evaluate the frequency of monoallelic expression for the candidate genes, we measured their allelic ratios of expression by RT-PCR followed by pyrosequencing in the newly derived NPC clones (65-245 clones/gene). The allelic expression ratio is defined as the proportion of mRNA expression from the Castaneus (Cast) allele over the total expression. A large number of clones analysed allowed assessment of the distribution of allelic expression ratios across clones for the 13 genes. Strikingly, we observed that the genes exhibit very different distribution profiles (Fig. 2). In order to decompose the distribution of the allelic expression ratio for each gene, we computed Gaussian mixture models to fit the data (see Methods), as this approach can reveal subpopulations of clones and putative allelic expression states. The number of populations for each gene was estimated using the Bayesian Information Criterion (BIC) (Supplementary Fig. 2). We used bootstrapping (see Methods) to assess both confidence in the number of populations (Supplementary Table 2) and in each Gaussian mixture model component (Supplementary Table 3). This analysis shows that our sample size provides us sufficient power to detect distinct populations. For each gene, we identified one or several subpopulations, defined by a specific mean and variance of the allelic expression ratio (Fig. 2 and Supplementary Table 3). Overall, genes display distinct modalities of allelic expression.

For Eya3, App, Ptk $2 b$ or Snca, we found a single population of clones (Fig. 2-top panels), however with different variances. The biallelic control Eya3 displays a narrow variance, and a mean allelic ratio of $41 \%$ (\% Cast) (indicative of a slight genetic bias towards expression of the 129 allele). App is biallelic in all the 83 NPC clones tested. Snca and Ptk2b both show a large variance, with the presence of clones showing extreme allelic expression ratios.

For Cnrip1, Eya1, Eya2 and Kcnq2, at least two subpopulations could be identified (Supplementary Fig. 2 and Supplementary Table 2), showing that these genes have more than one modality of allelic expression, each of them with a different variance and degree of allelic expression imbalance (Fig. 2-middle panels). For example, Cnrip1 is frequently monoallelically expressed from the Cast allele (30\% of clones) (Supplementary Table 3 ), whereas very rare clones display monoallelic expression from the 129 alleles, suggestive of an additional strong genetic bias. Eyal on the other hand does not fully exhibit monoallelic expression in any of the clones analysed, but displays two clear subpopulations, biased towards either the 129 or the Cast allele. This observation is unexpected: some genes (such as Eyal or Kcnq2) do not necessarily show any monoallelic population, but they do show two modalities of expression with different degrees of allelic expression imbalance. They are neither biallelic nor RME genes, but rather display RAExI.

The last group of genes, which includes A2m, Acyp2, Bag3, Eya4 and Grik2, was analysed using additional clones from differentiation experiments 4 to 6 , as they appeared to have more complex distributions (Fig. 2 -bottom panels and Supplementary Fig. 2). These genes show three or more modalities of expression, with gene-specific subpopulations (Supplementary Table 3). They display distinct monoallelic populations in both directions, comprising $18-44 \%$ of the clones analysed (Supplementary Table 3), as well as biallelic or biased populations. Thus, $A 2 m$, Acyp2, Bag3, Eya4 and Grik2 can be considered as RME, RME being a subtype of RAExI. The random monoallelic expression of these five genes is of particular interest as they have been associated with various diseases (Table 1), such as Acyp2 with cancer $^{22}, A 2 m$ with Alzheimer's disease ${ }^{23}$, Bag3 with myopathy ${ }^{24}$, Eya4 with deafness ${ }^{25}$ and Grik2 with epilepsy ${ }^{26}$. Acyp2 and Bag3 show the highest proportion of monoallelically-expressing NPC clones, with small variance, and thus represent good candidates for further exploring the features of allele-specific expression.

RAExI is established and maintained during differentiation. To determine whether the different states of allelic expression observed in NPCs arise during differentiation or are already present in ESCs, we established 18 ESC clones from the hybrid female ESC line and measured the allele-specific expression of Acyp2 and Bag3 using RT-PCR and pyrosequencing. Both genes were found to be biallelically expressed in all ESC clones tested (Fig. 3a, left panels), in contrast to NPCs, as shown using cumulative frequency distributions of the allelic expression ratios 

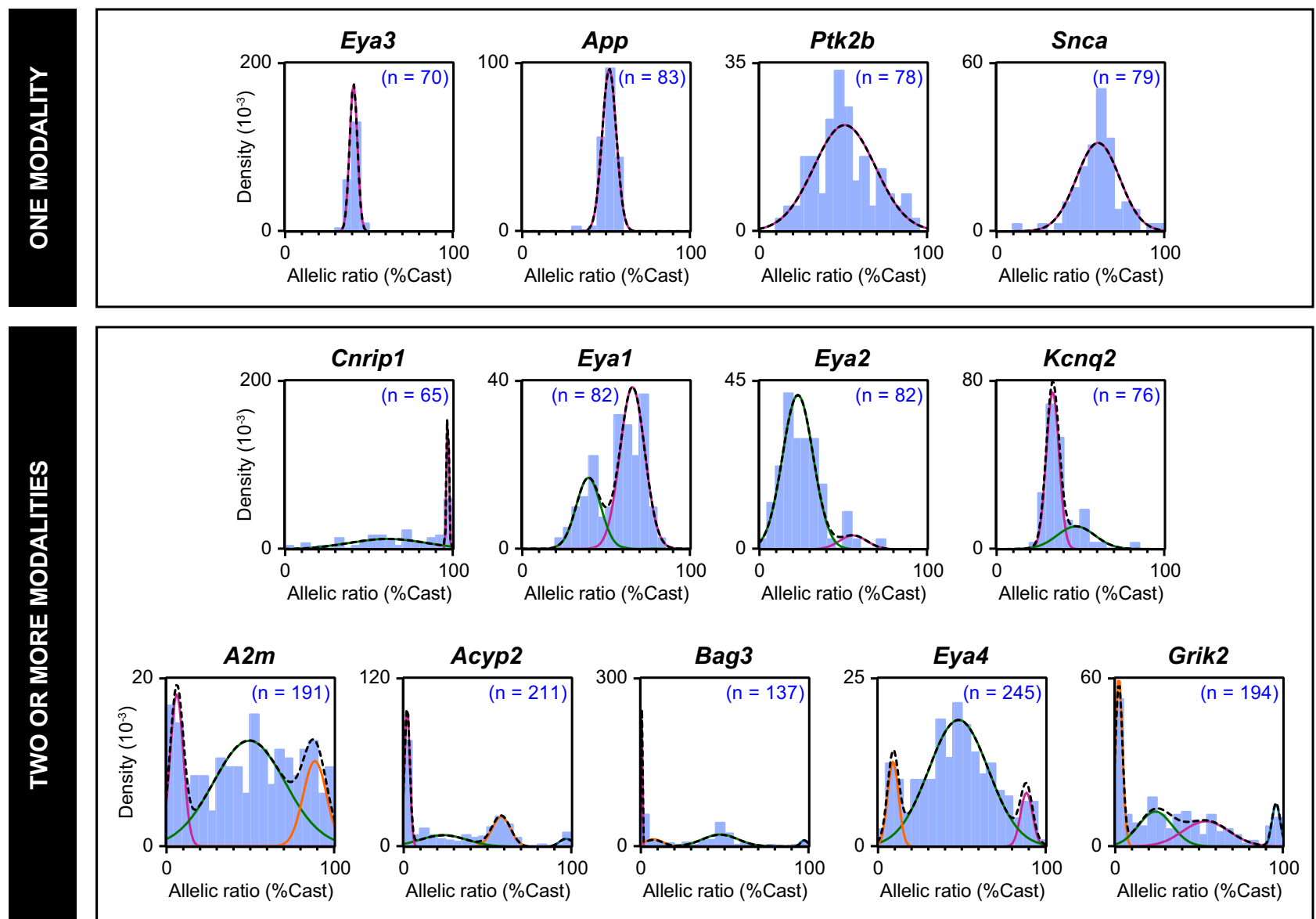

$$
\begin{array}{lll}
\text { Key } & \text { Observed distribution } \\
& \ldots & \text { Fitted mixture of Gaussians (components }-1-2-3-4 \text { ) }
\end{array}
$$

Fig. 2 Gene-specific modalities of allelic expression. The distributions of allelic expression ratios in NPC clones are represented as density histograms for the 13 genes of interest. $n$ is the number of NPC clones analysed for each gene. The observed distributions are modelled with Gaussian mixtures. The genes were classified according to the number of subpopulations, computed using the BIC criterion (Supplementary Fig. 2).

in both cell types (Fig. 3a, right panels). This indicates that allelespecific expression is established during differentiation.

We also evaluated the stability of allele-specific expression following further differentiation of NPCs to astrocytes. We previously showed that the RME pattern of six genes is maintained over cell passaging, and following differentiation of two independent NPC clones to astrocytes ${ }^{11}$. Here, we expand this analysis by performing allele-specific RNA-seq of five NPC clones after differentiation into astrocytes (Supplementary Fig. 3). We observed that the allelic expression ratios for the genes previously listed as RME in NPCs ${ }^{11}$ are globally stable following differentiation (Fig. 3b). Likewise, the allelic expression ratios of our RAExI genes appear stable (Fig. 3c). Overall, these analyses confirm and expand previous observations ${ }^{10,11}$ that allelic expression imbalance is established during differentiation of ESCs to NPCs and stably maintained once established.

RAExI gives rise to stochastic diversity. We next explored whether the allelic choices established during differentiation of ESCs to NPCs are in any way coordinated for multiple independent genes. This might occur if a common mechanism were involved in establishing or maintaining allele-specific expression of different genes, in particular for genes belonging to the same family (such as the Eya gene family). In order to reveal any potential concomitance in the allelic choices of the different genes, we performed hierarchical clustering of the allelic expression ratios for our 13 selected genes in the 44 NPC clones for which we had pyrosequencing data for all genes (Fig. 4a). We found that each NPC clone has a unique combination of allelic expression ratios for different genes and that none of the genes were coordinated, indicating that genes make allelic decisions independently of each other.

The NPC clones in this study were generated following six independent differentiation experiments of two ES cell lines. In order to assess whether the allelic choice for a given gene is deterministic, we compared the outcomes of all experiments. We observed that the allelic choices can be highly variable between differentiation experiments, notably for $A 2 m$, Acyp2, Bag3, Cnrip1, Eya2 and Eya4 (Fig. 4b and Supplementary Fig. 4). These observations indicate that the allelic choice is more consistent with a stochastic establishment during differentiation. The multiple combinations of allelic expression patterns that appear during differentiation therefore give rise to an unpredictable high level of cellular diversity.

RME is associated with reduced gene expression dosage. We next explored the link between monoallelic expression and gene dosage by measuring the expression levels by RT-qPCR for the 
a

Acyp2
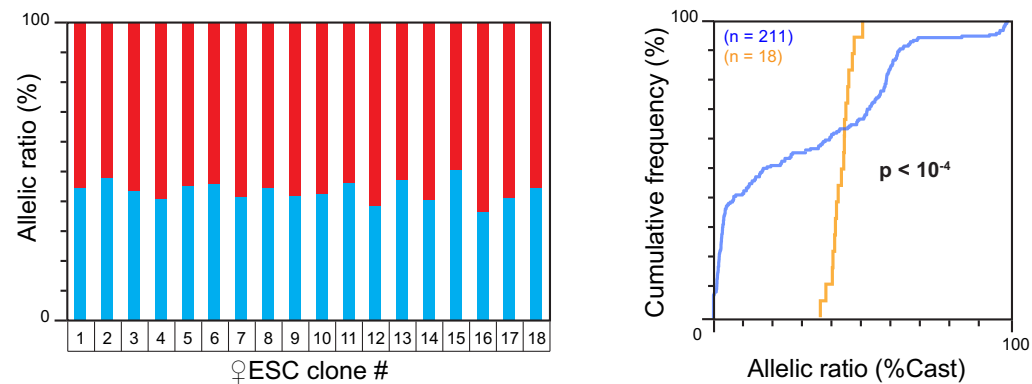

Bag3
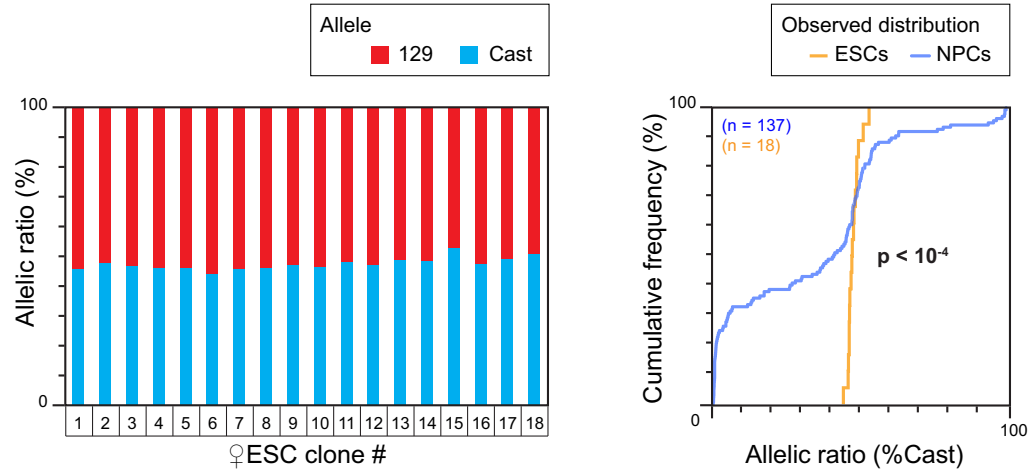

b

C
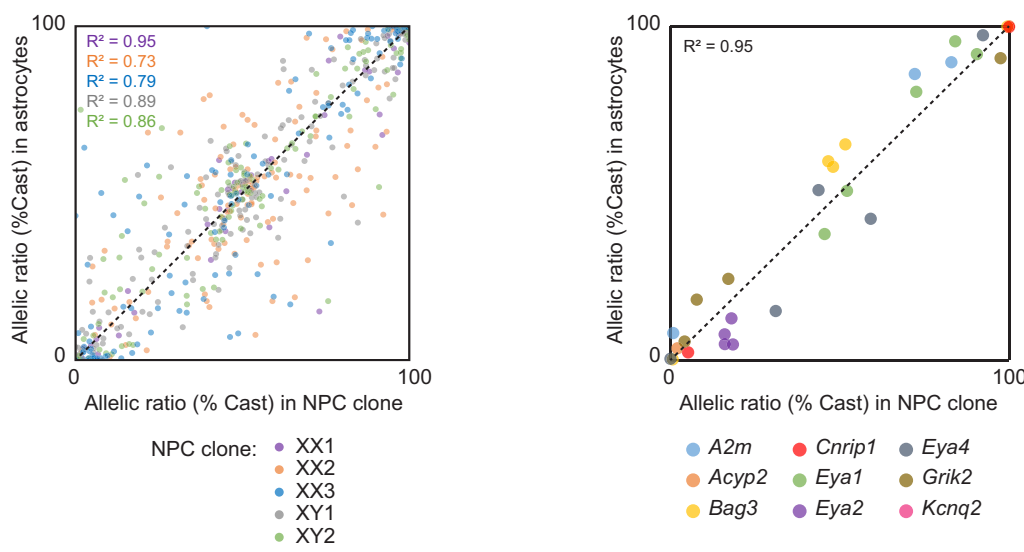

Fig. 3 Allele-specific expression is established during differentiation and then maintained. a Analysis by RT-PCR followed by pyrosequencing of Acyp2 and Bag3 allelic expression ratio in 18 clones generated from the F1-21.6 ESC line (left panels). Comparison of the cumulative distributions of allelic expression ratios in ESCs and NPCs (Kolmogorov-Smirnov tests, right panels). $n$ is the number of clones analyzed. b Comparison of the allelic expression ratios of the genes previously categorised as RME in NPCs ${ }^{11}$ and after differentiation into astrocytes, for five NPC clones, measured by allele-specific RNAseq. The number of assessable genes per clone (values with less than 20 reads were excluded) is $n_{X X 1}=75, n_{X X 2}=146, n_{X X 3}=121, n_{X Y 1}=150, n_{X Y 2}=137$. Pearson correlation coefficients were calculated for each NPC clone. c Comparison of the allelic expression ratio for the nine genes, which showed several modalities of expression, in five NPC clones and after differentiation into astrocytes.

RME genes Acyp2, Bag3 and the biallelic gene Eya3 in all NPC clones. While the biallelic control Eya3 shows similar expression levels in all clones, Acyp2 and Bag3 could be divided in two main groups, showing either high or low expression levels (Fig. 5a). Interestingly, we found that there are fewer monoallelicallyexpressing clones in those with low expression than in the group of clones showing high expression, indicating that monoallelic expression is not necessarily associated with low expression. Moreover, in clones with high expression levels, we found that the quantity of mRNA is reduced by half in monoallelic compared to biallelic clones. This impact on dosage is also observed at the protein level for $\mathrm{Bag} 3$, as we found a highly significant linear correlation between mRNA and protein levels (Fig. 5b and
Supplementary Fig. 5). BAG3 is a multifunctional co-chaperone protein that regulates diverse biological processes such as apoptosis and autophagy. It is a dosage-sensitive gene as its overexpression has been shown to promote apoptosis ${ }^{27}$, while its downregulation attenuates it ${ }^{28}$. RME appears to be a way to down-regulate protein levels of some genes and thus could have consequences on cellular functions.

Cell-type specificity of RME. We next addressed the potential impact of RME in vivo for some of our candidate genes, at the adult stage. We established a protocol (see Methods), which allows the detection of nascent RNA in the adult brain, known for 
a

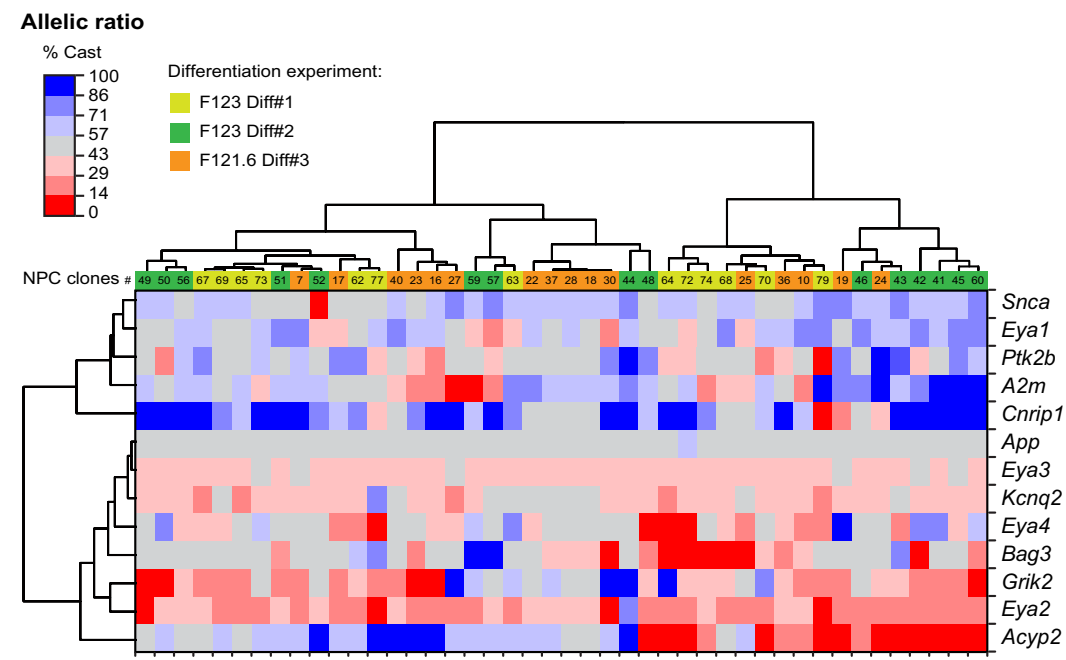

b

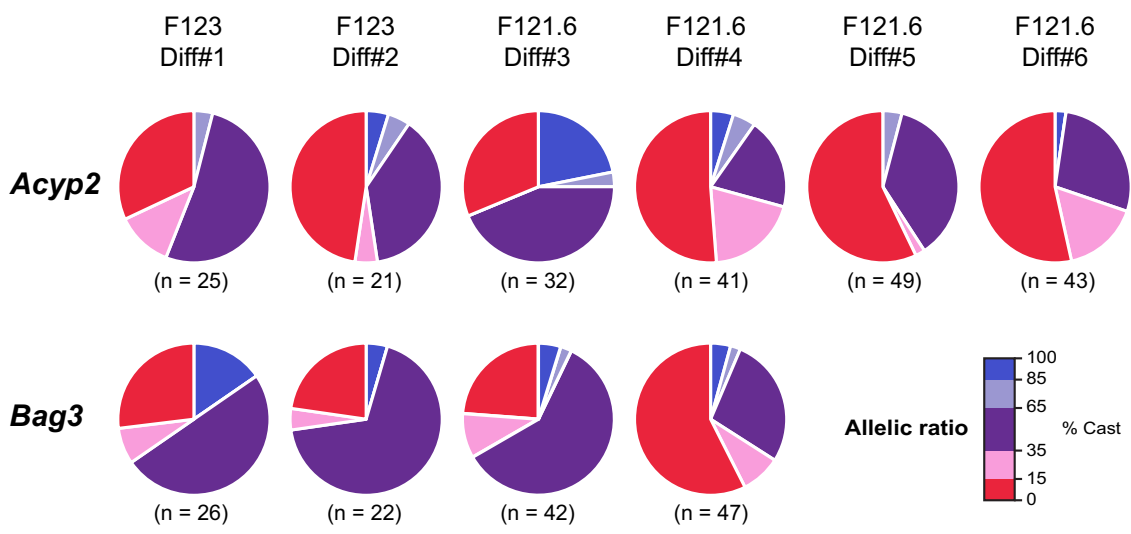

Fig. 4 Allelic imbalance generates cellular diversity. a Hierarchical clustering heatmap of allelic expression ratios for 13 genes in 44 NPC clones. b Proportions of NPC clones in each category of allelic expression status for Acyp2 and Bag3 for six independent differentiation experiments. $n$ is the number of clones analysed for each gene and each differentiation.

accumulating age-related autofluorescent molecules and lipofuscin-like pigments. In vivo analyses are generally limited by problems arising from cellular heterogeneity, transcriptional bursts and detection efficiency. We, therefore, applied a statistical test to evaluate whether or not we could rule out the possibility that the number of monoallelic cells observed by RNA-FISH would simply be a consequence of transcriptional bursts or a lack of detection (see Methods). To perform this analysis, both alleles must be genetically identical (inbred background), and the cells analysed must be homogeneous (i.e. same cell type, with the same kinetics of transcriptional bursts and the same probability to detect an expressed allele for a given gene).

In the brain, different cell types are usually intermingled, except in some specific regions, such as the hippocampus, where the somas of homogeneous cell types are tightly packed, making it an ideal model to investigate RME in vivo. Among the genes studied here, App and Grik2 are specifically relevant to study in this region, as they are respectively associated with Alzheimer's disease and epilepsy (Table 1), two pathologies that affect the hippocampus. We performed nascent RNA-FISH for App and Grik2 on hippocampal sections of adult inbred (C57Bl/6 J) mice and determined the percentage of cells showing monoallelic expression in the pyramidal neurons of the CA1 and CA3 regions. We observed that Grik2 is detected as monoallelic in 33\% of cells on average in CA1, unlike in the neighbouring CA3 region where Grik2 is biallelic, whereas App is biallelically expressed in both CA1 and CA3 neurons (Fig. 6). The percentage of CA1 neurons expressing Grik2 monoallelically is similar for the three animals analysed, and the observed distribution is significantly different from what would be expected from a biallelic gene showing bursty expression or with incomplete detection (see Methods). This indicates the presence of stable monoallelic expression in vivo. These results parallel the situation in NPCs, where App is biallelic and Grik2 shows RME (with $39 \%$ of the clones showing monoallelic expression) (Supplementary Table 3). Even though App was found to be monoallelic in lymphoblastoid cell lines ${ }^{6}$, it is not monoallelic in our study, indicating that this dosage-sensitive gene is not regulated in an allele-specific manner in hippocampal mouse neurons or NPCs. Monoallelic expression of Grik2 appears to be cell-type specific in the hippocampus in vivo. Interestingly, these findings are consistent with the observation that Grik2 tends to be more highly expressed in $\mathrm{CA}^{29}$. Moreover, it has been shown that increased expression in CA1 pyramidal cells is deleterious as it can trigger seizures ${ }^{30}$. Thus, RME may contribute to the regulation of gene dosage both in vitro and potentially in vivo. 

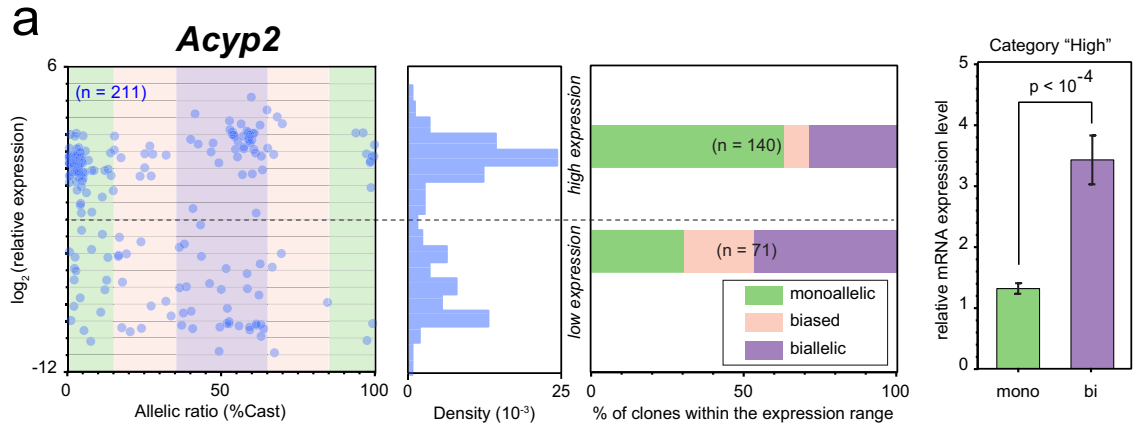

Bag3
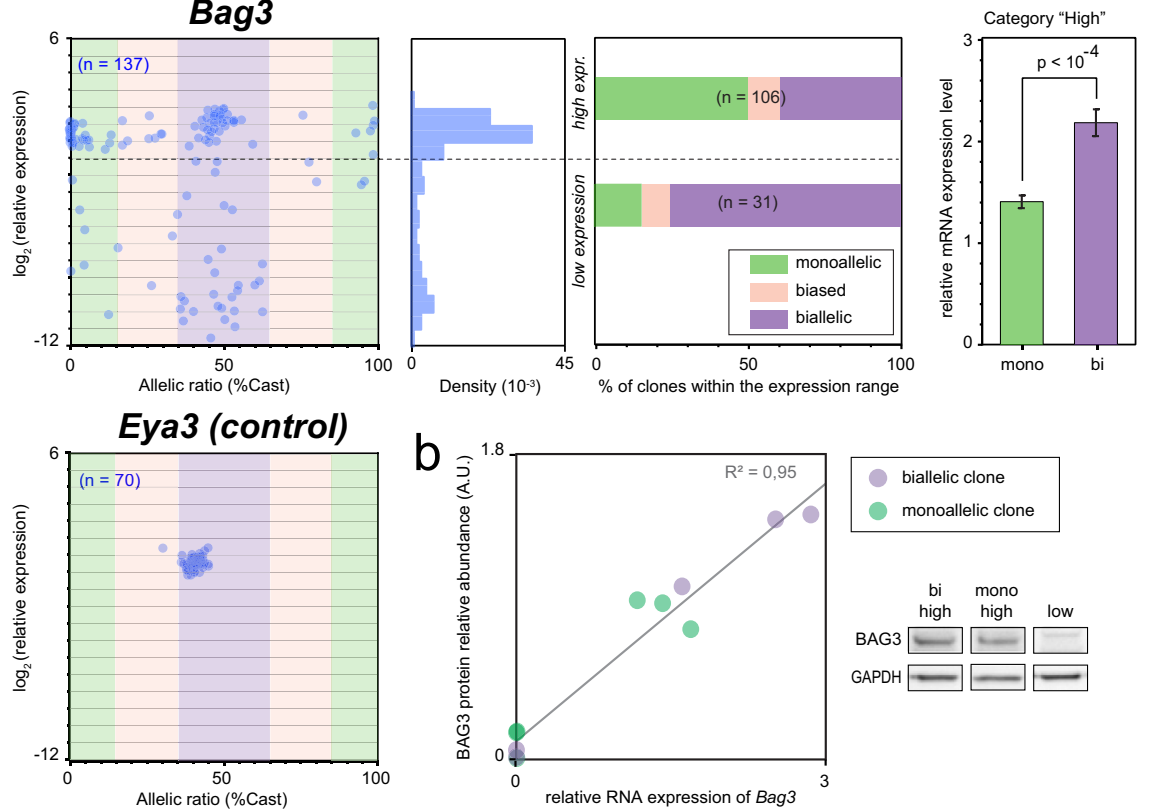

Fig. 5 Random monoallelic expression is associated with reduced gene dosage. a Comparison of the allelic expression ratio and mRNA expression levels in NPC clones (measured by qPCR), for Acyp2, Bag3 and Eya3 ( $n$ is the number of clones analysed for each gene; left panels). The density histograms show the distribution of expression levels; the dashed lines indicate the threshold used to categorise high or low expressing clones (middle left panels).

Proportion of clones in each allelic category for clones showing high or low expression levels (middle right); allelic categories: monoallelic [ratio < $15 \%$ or $>85 \%$ ], biased [ratio $15-35 \%$ or $65-85 \%$ ], biallelic [ratio $35-65 \%$ ]; $n$ is the total number of clones. mRNA levels in monoallelic clones compared to biallelic clones (excluding the clones with low expression levels -right panels). Data were means \pm SEM; two-tailed unpaired $t$-tests, Acyp2 $t=6.5 \mathrm{df}=131$, Bag 3 $t=5.6 \mathrm{df}=92$. b BAG3 relative protein levels measured by western blot (normalised to GAPDH levels), compared to the relative RNA abundance measured by RT-qPCR, for a selection of 11 NPC clones. Linear regression $F_{(1,9)}=176, p<10^{-4}$. A western blot image of the Bag3 full-length form is shown for three representative NPC clones. Full original blot images are provided in Supplementary Fig. 5.

Local regulation of RME. To explore how allele-specific expression could be regulated, we examined whether neighbouring genes to loci showing RME (A2m, Acyp2, Bag3, Eya4, Grik2, Fig. 2) also showed RME in the same clones, or some degree of RAExI. Common trans-acting factors could control several genes sharing cis-elements within a genomic region. We used allele-specific RNA-seq datasets from 16 NPC clones (nine previously published and seven additional clones from this study - Supplementary Table 4) $)^{11,31}$ and compared the distribution of allelic expression ratios for the Acyp2, A2m, Bag3, Eya4 and Grik2 genes, together with eight neighbouring genes located upstream and downstream (Fig. $7 \mathrm{a}$ and Supplementary Fig. 6a). We found that neighbouring genes generally show biallelic expression, with a few exceptions that display uncorrelated allelic imbalance (Apobec1, Slc18b1). This indicates that the RME is not regulated within a domain but is rather an intrinsic property of each gene, in agreement with previous observations ${ }^{11,13}$.

We then aimed at identifying the regulatory regions controlling allelic imbalance expression of the genes validated as RME ( $A 2 m$, Acyp2, Bag3, Eya4 and Grik2). We used allele-specific ATAC-seq datasets available for 13 NPC clones (assay for transposaseaccessible chromatin) ${ }^{13}$, and RNA-seq for the same clones (seven previously published and six newly sequenced-Supplementary Table 4). In a previous study ${ }^{13}$, in seven NPC clones for which both RNA-seq and ATAC-seq data were available, RME genes were found to be associated with random monoallelically accessible elements located mostly at promoters. However, this study was carried out in a genome-wide manner without investigating our individual RME genes of interest.

We analysed all peaks of accessible chromatin with allelespecific information within a $6 \mathrm{Mb}$ region surrounding each gene (see Methods). For each peak, we determined whether the allelespecific chromatin accessibility correlated with allele-specific expression, by linear regression (Fig. 7b -left panels and Supplementary Fig. 6b, c -left panels). Even though not all peaks had allelic information, we found a genomic region that shows a significant correlation with allelic expression for A2m, Acyp2 Bag3 and Grik2, located in each case at the transcription start site (TSS) (Fig. 7b and Supplementary Fig. 6c, middle and right panels). This is indicative of local regulation of RME, in line with 

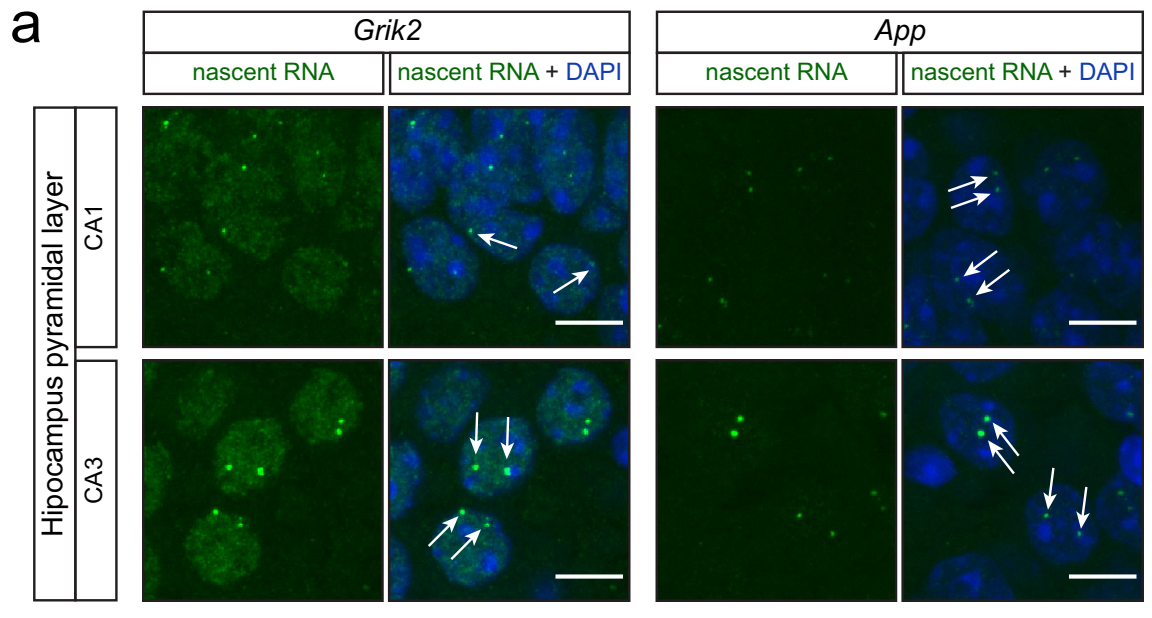

b

Grik2

App
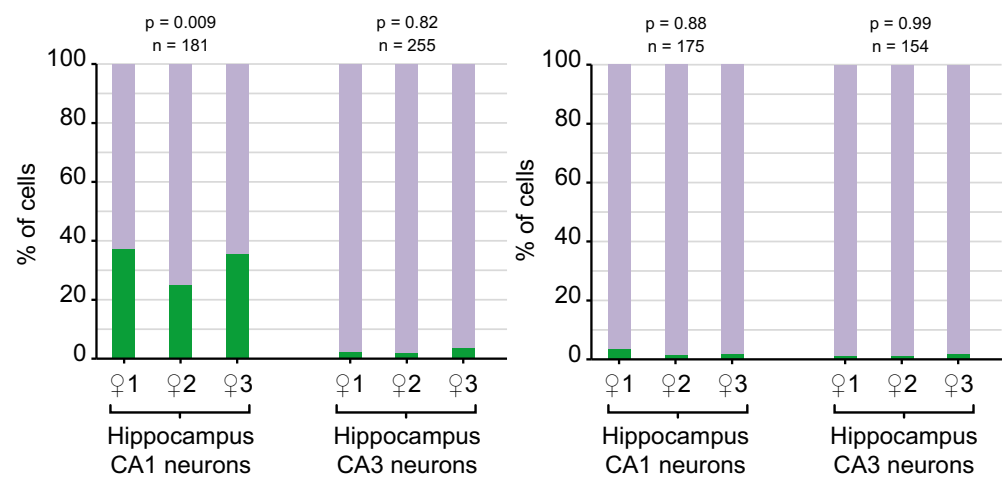

number of RNA-FISH

pinpoints detected

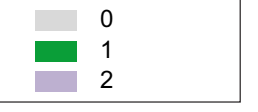

Fig. 6 Monoallelic expression is cell-type specific in vivo. a Z-stack of confocal images showing representative cells with nascent RNA-FISH signals for Grik2 and App, in hippocampal sections of adult inbred mice. Scale bar $10 \mu \mathrm{m}$. b Quantification of nascent RNA-FISH in pyramidal neurons of CA1 and CA3, in the three female inbred mice analysed. Chi-squared test (see Methods); $n$ is the number of cells analysed for each gene and each region.

the observation that random monoallelically accessible elements are enriched at promoters ${ }^{31}$.

Epigenetic maintenance of RME. Acyp2 and Bag3 showed the highest correlation at the TSS between chromatin accessibility and expression (Fig. 7b). The allele-specific accessible region at the TSS overlaps with a well-defined CpG island (CGI). We, therefore, measured DNA methylation levels in 20 representative NPC clones and in ESCs using Sequenom analysis of bisulfitetreated DNA. We found that CGI are hypermethylated in clones where the gene is silenced or lowly expressed, hypomethylated in biallelically-expressing clones and in ESCs, and show an intermediate level of methylation in clones with monoallelic expression (Fig. 7c). This suggests that the alleles that are lowly expressed become methylated during differentiation and that DNA methylation correlates with expression levels, rather than directly with the allelic expression status.

To further investigate the role of DNA methylation, as well as other epigenetic mechanisms in the maintenance of RME patterns in NPCs, we designed a screen using a library composed of 181 drugs targeting epigenetic modifications or pathways (Supplementary Table 5). We applied the screen to Bag3 as a proof of principle, as it is a particularly interesting target for allele-specific regulation, due to its multiple functions, its dosage sensitivity and its implications in disease. First, we measured the impact of these molecules on Bag3 allelic expression in two selected female NPC clones (\#31 and \#84, expressing Bag3 from the 129 allele or the Cast allele, respectively), after two days of treatment at a standard concentration of $10 \mu \mathrm{M}$ (see Methods). We identified several epidrugs that induce an increase in the ratio of expression of the silent allele in both clones (Fig. 8a). A secondary test was conducted using the 11 hits that showed the strongest shift on both NPC clones, on a higher number of cells from NPC clone \#84 (Fig. 8b). This analysis confirmed that treatment with the DNA methyltransferase inhibitor Decitabine, as well as two histone deacetylase (HDAC) inhibitors (Dacinostat and CUDC-101) significantly increase the Bag3 ratio of expression of the silent allele. These drugs inhibit transcriptional repression mechanisms. In order to refine the concentration, we performed a dose-response of Decitabine and Dacinostat, with fresh compounds. The two drugs showed a dose-dependent effect, and reached a maximal effect at $10 \mu \mathrm{M}$ (Fig. 8c), with an EC50 of 1.6 and $0.9 \mu \mathrm{M}$, for Decitabine and Dacinostat respectively. Finally, we confirmed their effect on both clones, with a $10 \mu \mathrm{M}$ treatment reduced to $24 \mathrm{~h}$ (Fig. $8 \mathrm{~d}$ ). These analyses show that the silent allele of Bag3 can be reactivated, and thus it importantly shows that RME is epigenetic, rather than caused by genetic aberrations or gene rearrangements. The maintenance of monoallelic expression of Bag3 in NPCs relies on a combination of epigenetic modifications, including DNA methylation and histone deacetylation, which are actively involved in the inheritance of the transcriptional state. The inhibition of such pathways can be used to reexpress the silent allele, offering perspectives for therapeutic strategies for RME genes associated with autosomal dominant disorders. 

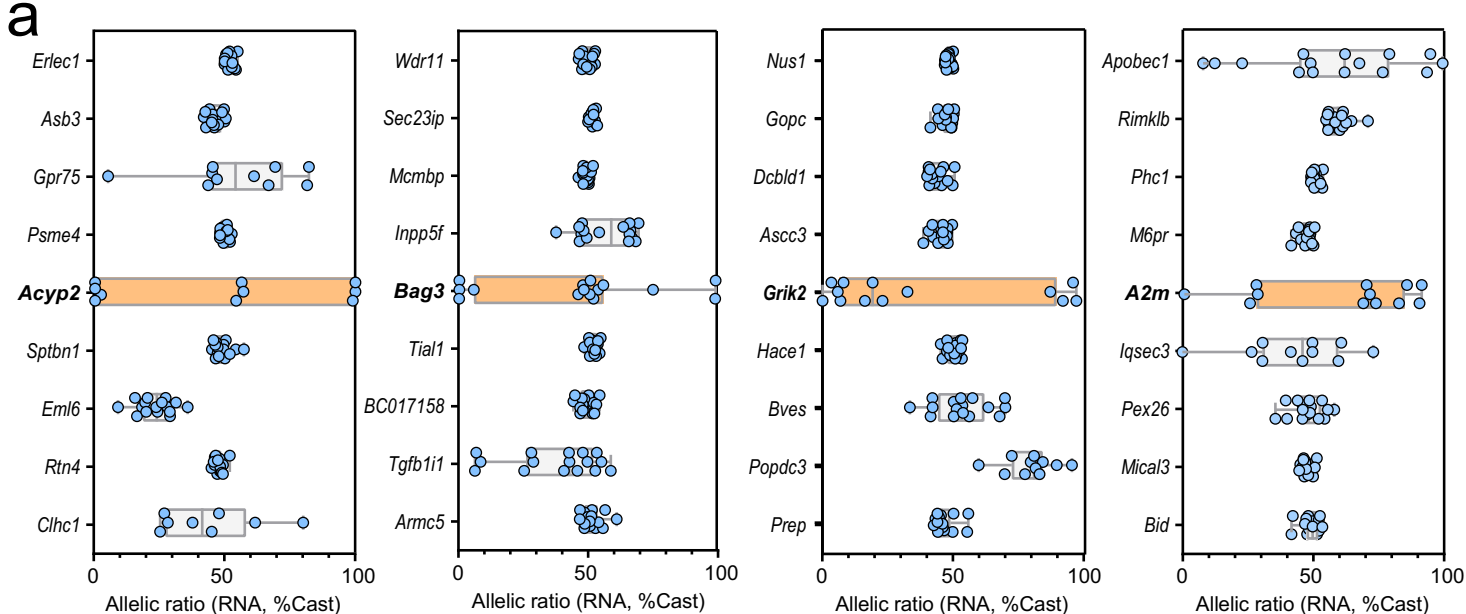

\section{b Acyp2}
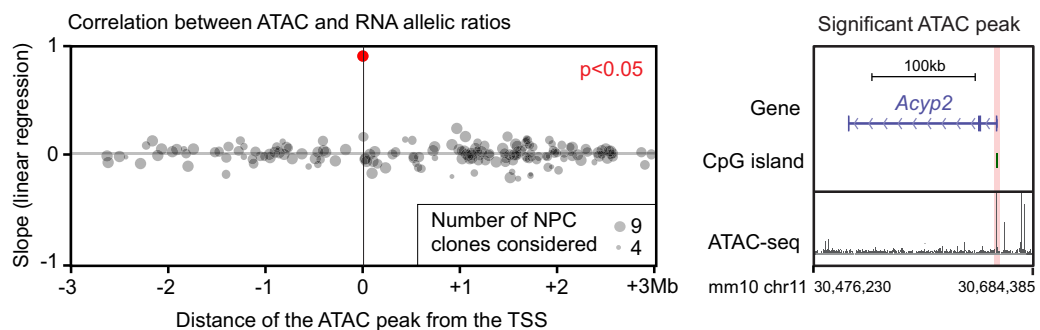

\section{Bag3}

Correlation between ATAC and RNA allelic ratios
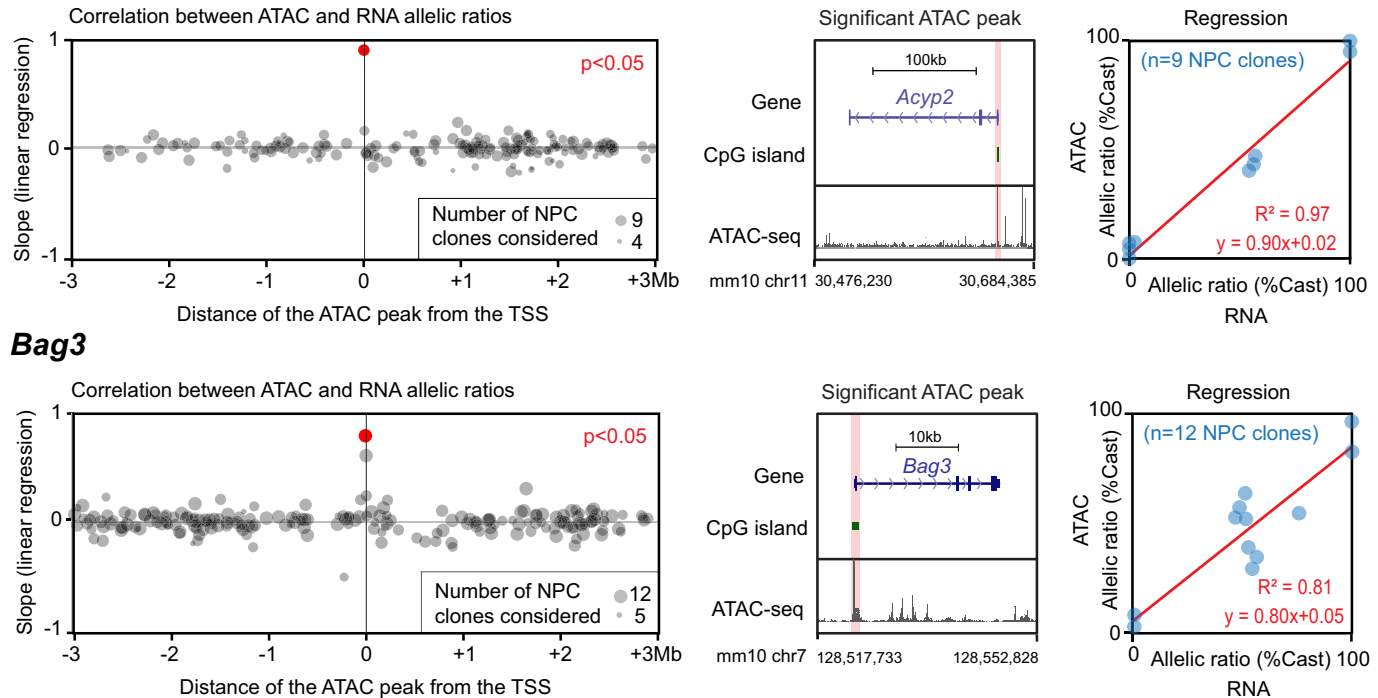

C Acyp2

Bag3
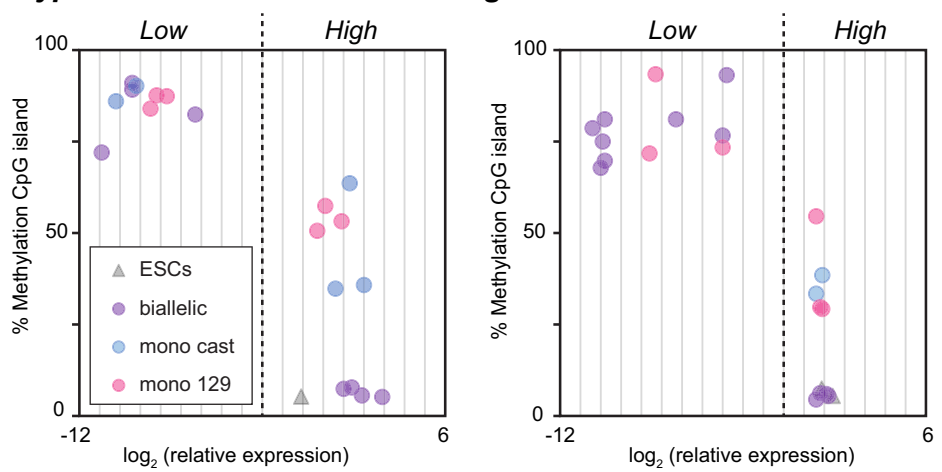

Fig. 7 Allelic imbalance is regulated locally. a Comparison of the distribution of allelic expression ratios for Acyp2, Bag3, Grik2 and A2m, with four upstream and four downstream neighbouring genes, measured by allele-specific RNA-seq in 16 NPC clones. Boxplot elements: centre line represents the median, box bounds represent the 25th and 75th percentiles, whiskers represent the minimum and maximum. The orange colour highlights the boxplot plot of the RME gene of interest. $\mathbf{b}$ Identification of the genomic region where allele-specific accessibility correlates best with expression, by linear regression between the ATAC-seq allelic accessibility ratio and RNA-seq allelic expression ratio, for ATAC-seq peaks (containing at least one SNP) within a region of \pm 3 megabases around the TSS. P value adjusted with Bonferroni correction, number of ATAC peaks: 177/207 Acyp2/Bag3 (left panels). UCSC genome browser snapshot showing a representative ATAC-seq track along the gene, with highlighting of the ATAC-seq peak, located at the TSS, that correlates significantly with the RNA-seq (middle panels). Linear regression showing the correlation between the ATAC-seq allelic accessibility ratio and the RNA-seq allelic expression ratio at the TSS (right panels). ATAC-seq data and RNA-seq datasets are from 13 NPC clones. Acyp $2 F_{(1,7)}=227, p=1.3 \times 10^{-6} ; B a g 3$ $F_{(1,9)}=42, p=7.3 \times 10^{-5}$. c DNA methylation levels over the CpG island located at the TSS of Acyp2 and Bag3, measured by Sequenom bisulfite analysis in 21 representative NPC clones and the two ES cell lines, characterised by high or low expression levels and mono- or biallelic expression. 


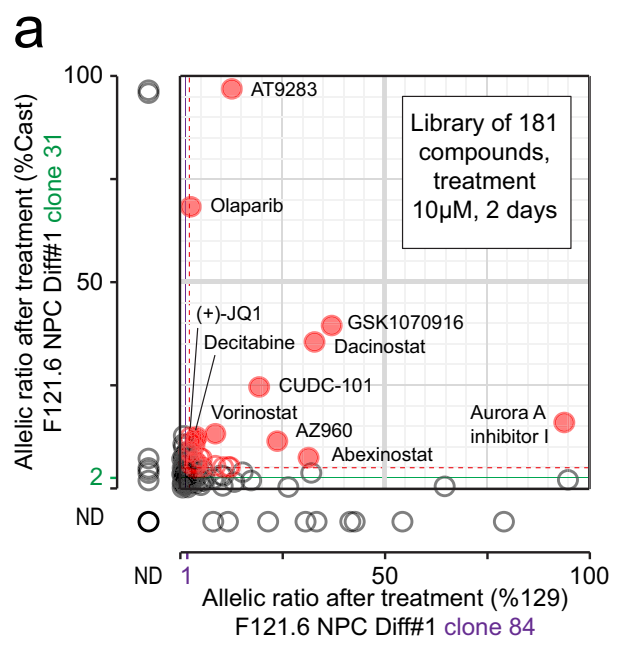

C

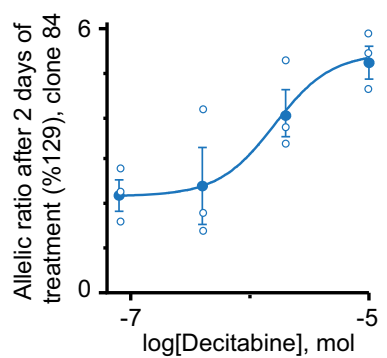

$\mathrm{b}$
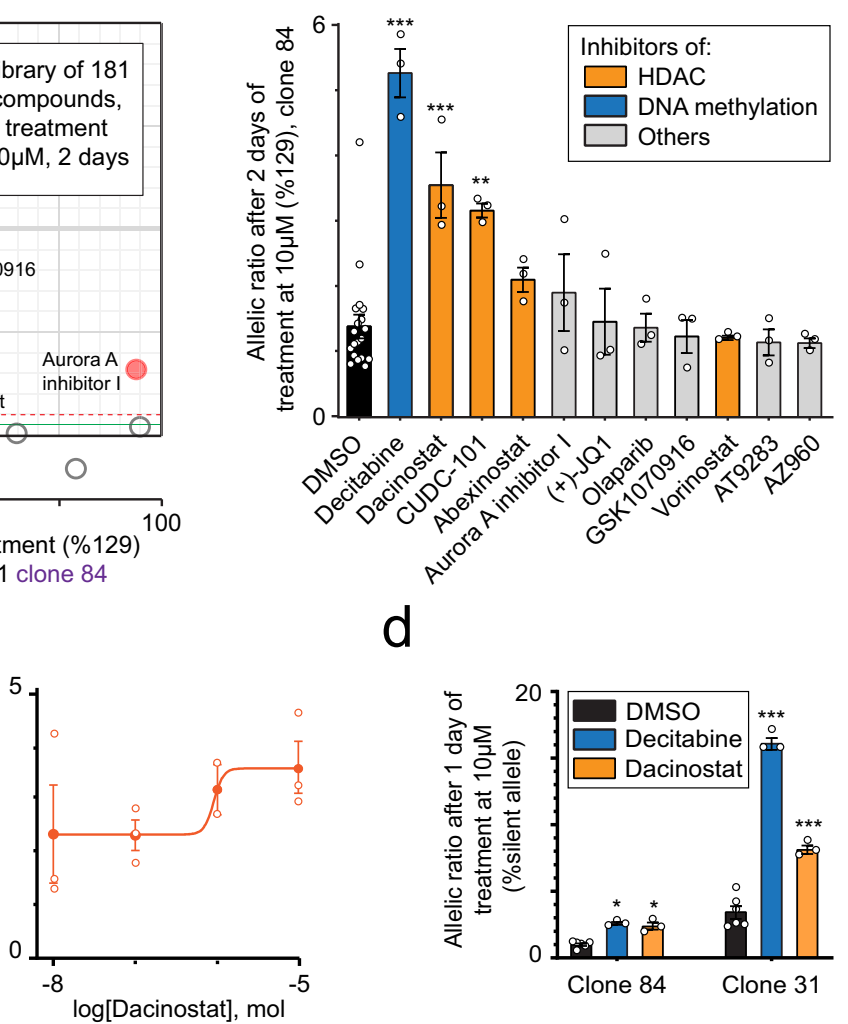

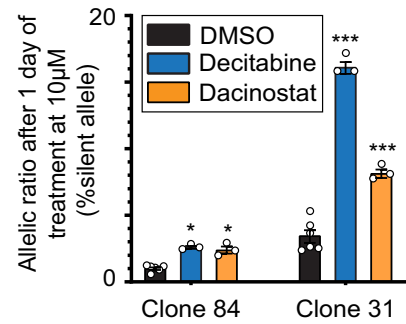

Fig. 8 Epigenetic maintenance of the monoallelic expression of Bag3. a Effect of treatment with an epidrug library $(2$ days at $10 \mu \mathrm{M})$ on the allelic expression ratio of two NPC clones (\#31 and \#84), measured by pyrosequencing following RT-PCR. Purple and green lines represent the mean of the 11 controls, dashed red lines are mean $+3 \times$ Stdev, red circles are compounds with an effect $>$ mean $+3 \times$ StDev for both clones. ND no data $\mathbf{b} V$ alidation of three compounds among the 11 best hits from (a) on NPC clone \#84 (2 days of treatment at $10 \mu \mathrm{M}$ ). DMSO is used as the vehicle. Data were means \pm SEM $\left(n=3\right.$ replicates per condition). One-way ANOVA: treatment effect, $F_{(11,40)}=12, p<10^{-4}$. Dunnett's post hoc test: ${ }^{\star} p<0.05$, ${ }^{\star \star \star} p<10^{-4}$ (DMSO versus treatment). c Dose-response curve for Decitabine and Dacinostat for NPC clone \#84, after 2 days of treatment. Data were means \pm SEM ( $n=3$ replicates per condition). $\mathbf{d}$ Confirmation of the effect of Decitabine and Dacinostat on NPC clones \#31 and \#84, after 1 day of treatment at $10 \mu M$. DMSO is used as the vehicle. Data were means $\pm \operatorname{SEM}\left(n=3\right.$ replicates per condition). Two-way ANOVA: treatment effect, $F_{(2,18)}=200, p<10^{-4}$; clone effect $F_{(1,18)}=548$, $p<10^{-4}$; interaction, $F_{(2,18)}=120, p<10^{-4}$. Bonferroni post hoc test: ${ }^{\star} p<0.05,{ }^{\star \star \star} p<10^{-4}$ (DMSO versus treatment).

\section{Discussion}

The main goal of our study was to explore whether and how some autosomal genes are prone to persistent and stable monoallelic expression, by focusing on a subset of genes of interest. The analysis of a very high number $(>200)$ of NPC clones revealed that genes previously identified as RME actually display one or several modalities of allelic expression, with different degrees of allelic imbalance. These observations unexpectedly uncovered the existence of RAExI. In light of our findings, RME appears to be a specific case of RAExI.

In this study, we used NPCs to assess RAExI and we showed in vivo that this phenomenon can be cell-type specific. It will be important to expand the study of RAExI to different cell types and other genes to assess its extent, as this may be a broader phenomenon than anticipated. The examples of Eya3 and App demonstrate that some genes can be strictly biallelic. However, App was found to be RME in a different cell type ${ }^{6}$, reinforcing the idea that RAExI is cell-type specific. Biallelic genes in one cell type could show RAExI in another. Reciprocally, genes showing RAExI in NPCs could be biallelically expressed in a different context. Therefore, it appears particularly relevant in future studies to assess whether genes associated with autosomal dominant disorders have a RAExI pattern in specific tissues, because it could play a role in the aetiology of the disease in a noncanonical manner.
RAExI can be a means to regulate the differential allelic abundance of transcripts, which contributes to the control of gene expression levels in addition to other effects, such as genetic variation. Indeed, genetic polymorphisms at specific loci partly explain variations of gene expression levels (expression quantitative trait loci, eQTLs) ${ }^{32}$. Parent-specific single nucleotide polymorphisms (SNPs) within cis-eQTLs can account for the differential allelic abundance of transcripts, causing a permanent imbalance in expression ${ }^{33}$. RAExI then allows variability of the allelic imbalance, in a given genetic context. In this study, we have used a $129 / \mathrm{Sv}$ x Cast hybrid background in vitro, and thus the observed precise modalities reflect the effect of RAExI in this genetic background.

The investigation of RME and RAExI brings technical challenges, as their observation can be confounded with poor detection, genetic aberrations or transcriptional bursting ${ }^{14,17}$. In this study, we managed to overcome these limitations. First, we measured the allelic expression ratio in more than $10^{6}$ cells per clonal cell line, thus averaging out the effect of transcriptional bursts. Moreover, we analysed more than 200 independent NPC clones, which gave us high statistical power and shows that monoallelic expression is not scarce for the genes analysed (it can be present at frequencies $>40 \%$ ). We computed the distribution of the allelic expression ratios, establishing that our observations are not due to technical or biological noise. Furthermore, we ruled 
out the contribution of genetic aberrations, not only because of the high frequencies of monoallelic expression, but also by showing that the silent allele can be reexpressed for the RME gene Bag3 using epidrugs. Taken together, our data display robust evidence of RAExI in vitro.

In vivo analyses are more limited, given the challenge of identifying clonal populations (i.e. cells that originate from the clonal propagation of one cell, after the allelic choice has been established in that cell for the genes of interest). Although there have been some efforts in this direction ${ }^{6}$, in vivo analyses are generally performed on single cells and thus, without clonal information. Therefore, these analyses are intrinsically limited by the bursty nature of transcription, which generates transient monoallelic expression or allelic imbalance ${ }^{31,32}$ that can be confounded with stable RME. Previous studies have observed monoallelic expression in vivo by nascent RNA-FISH ${ }^{11,19}$, but have not excluded the possible contribution of transcriptional bursts. In this study, we developed an RNA-FISH protocol for the adult brain, with autofluorescence removal and optimised sensitivity. The implementation of a statistical approach that takes into account the probability of detection, applied to a homogenous cell type in an inbred background, allowed us to show that transcriptional bursts are not responsible for the observed monoallelism of the RME gene Grik2, and indicates that stable RME can occur in vivo. This has potentially large consequences in physiology and disease 5 . The presence of RME in vivo could notably generate some functionally nullisomic cells in a heterozygous context with a loss-of-function mutation. This, for example, is the case of the Cubulin gene, which shows mosaicism in the kidney ${ }^{34}$, but it could potentially concern many other genes.

In this study, we also investigated the physiological consequences of allelic expression modalities. We show that these expression patterns generate a wide diversity of gene expression, as all the clones have a unique combination of allelic expression ratios. This diversity could confer adaptability to the organism to environmental perturbations (during development and through life), as some cells could have an advantageous combination of allelic modalities ${ }^{5}$. In addition, we show that monoallelic expression impacts gene dosage, not only at the mRNA level, but also the protein level. The regulation of gene dosage during development could have important implications in cell fate decisions and differentiation, notably, if a dosage-sensitive transcription factor would play a role in differentiation. For example, it has been shown that the stochastic allelic expression changes of the gene Bcl11b, which encodes a factor controlling T-cell commitment, influence T-cell fate timing and development ${ }^{35}$. RME or RAExI could also be a mechanism to fine-tune the levels of expression of specific genes in a particular cell type, such as Grik2 which shows monoallelism in the CA1 pyramidal neurons, where it is known to be expressed at lower levels ${ }^{29}$. Taken together, we show that RME or RAExI could have some advantageous physiological consequences for an organism.

Despite their potentially major consequences in physiology and pathology, the mechanisms underlying RME are largely unexplored. We show that genes previously categorised as RME do not represent a single homogeneous class of genes, but rather display a wide range of possible allelic states, with some of them showing RAExI rather than RME. The mechanisms of establishment and maintenance of the allelic states are thus most likely gene-specific. However, the different genes analysed share common features. First, all genes show biallelic expression in more than half of the NPC clones, indicating that there is no primary feedback selection mechanism to ensure strict monoallelism, thus the expression of both alleles appears to be independent (in contrast to immunoglobulins or olfactory receptors ${ }^{36}$ ). The monoallelic expression might arise through stochastic silencing or activation of one allele and subsequent clonal propagation. The high frequencies of monoallelism $(>40 \%)$ nevertheless indicate that the establishment of RME is not a fortuitous and rare event. Furthermore, all genes investigated have different allelic expression states, which are consistent across different cell lines and differentiation experiments. Thus, expression states appear to be predetermined and gene-specific. This contrasts with the proportions of cells in each state, which vary from one differentiation experiment to another. This variation could be a secondary effect, as some cells might acquire a growth advantage depending on the combination of their allelic choices. Thus, the establishment of the allelic states, by its nature, is stochastic, and has an unpredictable outcome, among the numerous predetermined possibilities.

Our study addresses more specifically the mechanisms of maintenance of RME. In line with previous observations ${ }^{13}$, we find that the regulation of RME takes place locally at the TSS. Several studies have associated RME with the presence of H3K4me3 and H3K9me3 marks respectively on the active and inactive alleles ${ }^{10,13,37,38}$, not only at the TSS but also on the gene body. Here, we show for the RME genes Acyp2 and Bag3, that the differentially accessible region at the TSS coincides with a CpG island, which is initially unmethylated in ESCs and is methylated only when the allele is silenced or lowly expressed after differentiation to NPCs. Furthermore, we demonstrate the causality between DNA methylation and the maintenance of monoallelic expression of Bag3, as inhibition of DNA methyltransferases by Decitabine contributes to the derepression of the silent allele. Our epidrug screen also uncovers the role of histone deacetylation in the maintenance of silencing. Hence, using the Bag3 gene as a proof of principle, we showed that epigenetic modifications contribute to the maintenance of RME. As allelic expression modalities are gene-specific, RME and RAExI genes are likely to depend on specific mechanisms and combinations of epigenetic modifications. The present work should provide a useful set of tools for the future study of other RME and RAExI genes.

We previously found that genes classified as RME are often associated with autosomal dominant disorders in humans and haploinsufficiency in mice ${ }^{11}$. As we observed that the proportions of cells in each allelic state are highly variable for each differentiation experiment, this could contribute to the incomplete penetrance of the disease associated with these genes between individuals. Bag3, for example, is associated with autosomal dominant dilated cardiomyopathy with partial penetrance and has been described as haploinsufficient ${ }^{39,40}$. We showed that Bag3 monoallelic expression is maintained by a combination of epigenetic modifications found mostly at the promoter region of the gene. In the case of a loss-of-function heterozygous mutation on a gene expressed in a random monoallelic manner, reactivating the wild-type allele using epidrugs or epigenome editing tools could offer a therapeutic strategy for some autosomal dominant disorders. Alternatively, the mechanisms of monoallelic expression could be used to selectively silence a dominant-negative allele.

In conclusion, we have uncovered unexpected, various and complex modalities of allelic expression, which go beyond the concept of RME. These modalities of expression may have broad implications in physiology and disease, and represent potential therapeutic perspectives. However, given that each gene appears to show a specific set of expression modalities, it implies that genes should be considered individually rather than by class. We hope that the present work will raise awareness about the concept of epigenetically regulated allele-specific expression, and that it will be considered and investigated in the study of specific genes, for their role both in physiology and pathology, and despite technical challenges. 


\section{Methods}

Cell culture. The female F1-21.6 and male F1-23 mouse ESC lines were a kind gift from Prof. Joost Gribnau. Their genetic background is a hybrid between Mus musculus $(129 / \mathrm{Sv})$ and Mus musculus castaneus (Cast/EiJ). ESCs were grown on mitomycin C-inactivated MEFs in Dulbecco's Modified Eagle Medium (DMEM) supplemented with foetal bovine serum $15 \%, \beta$-mercaptoethanol $0.1 \mathrm{mM}$ and leukaemia inhibitory factor $1000 \mathrm{U} / \mathrm{mL}$, at $37^{\circ} \mathrm{C}$ with $8 \% \mathrm{CO}_{2}$

ESCs were differentiated into NPCs as previously described ${ }^{11,21}$. Briefly, for each experiment, $1 \times 10^{6} \mathrm{ESCs}$ were plated in a petri dish coated with $0.1 \%$ gelatin, in N2B27 medium. After 7 days, cells were dissociated and $3 \times 10^{6}$ cells were plated in a bacterial petri dish in N2B27 medium supplemented with EGF $10 \mathrm{ng} / \mathrm{mL}$ (Peprotech) and FGF2 $10 \mathrm{ng} / \mathrm{mL}$ (R\&D systems), for 3 days. Neurospheres were then transferred in a gelatin-coated petri dish, in the same medium, for them to attach and the NPCs to expand. NPC clones were generated following manual colony picking after limited dilution of the NPC population. Clones were individually expanded and then harvested for RNA, DNA or protein extraction. NPC clones were differentiated in astrocytes after transfer in N2B27 medium supplemented with $1 \mathrm{ng} / \mathrm{mL}$ of FGF2 and $10 \mathrm{ng} / \mathrm{mL}$ of BMP4 (Systems) for $48 \mathrm{~h}$, as previously described ${ }^{11}$.

RNA extraction, reverse transcription, qPCR and pyrosequencing. Total RNAs were extracted from NPC cell pellets using the Qiagen RNeasy plus mini kit and treated with DNasel using the RNase-free DNase set (Qiagen), following the manufacturer's instructions. Total RNAs $(1 \mu \mathrm{g})$ were reverse-transcribed into cDNAs using random primers and SuperScript III reverse transcriptase (Invitrogen) for $1 \mathrm{~h}$ at $50^{\circ} \mathrm{C}$. Quantitative PCR (qPCR) was performed using Power SybrGreen PCR master mix on a ViiA 7 real-time PCR system (Applied Biosystems). The expression of genes was normalised to three housekeeping genes (Gapdh, B2m, Rrrm2). Clones were classified into 'low expression' and 'high expression' using an arbitrary threshold (indicated in Fig. 5). For pyrosequencing, PCR and sequencing primers were designed using the PyroMark Assay Design software (Qiagen). All successfully amplified PCR products were analysed on a PyroMark Q24 (Qiagen). All primer sequences can be found in Supplementary Tables 6 and 7.

Definition and classification of allelic ratios. The allelic expression (for RNAseq) or accessibility (for ATAC-seq) ratio was defined as the percentage of reads from the Castaneus allele. For sequencing data: (number of reads on the Cast allele)/(number of reads on the Cast allele + number of reads on the 129 allele). For pyrosequencing data: (abundance of sequences from the Cast allele)/(abundance of sequences from the Cast allele + abundance of sequences from the 129 allele). The ratios range from $0 \%$ to $100 \%$, corresponding respectively to exclusive monoallelic expression/accessibility from the maternal (129/Sv) allele or the paternal (Cast) allele for a given gene. The allelic categories (for Fig. 5a) are defined as follows: monoallelic [ratio $<15 \%$ or $>85 \%$ ], biased [ratio $15-35 \%$ or $65-85 \%$ ] and biallelic [35-65\%].

Analysis of allelic expression ratio distributions. The Gaussian mixture models were computed using the mclust package (version 5.4.7) ${ }^{41}$ of R, fitted via the EM algorithm. The optimal number of components was selected as the one maximising the BIC (Supplementary Fig. 2). To ascertain that the sample size was sufficient to identify population mixtures with distinct allelic ratios, we further conducted likelihood-ratio tests (LRT) using a bootstrap approach (with 1000 replications) to evaluate the null distribution and compute $p$ values $^{42}$ (Supplementary Table 2), using the mclustBootstrapLRT function of the mclust package. We further used a bootstrap approach (1000 replications again) to calculate $95 \%$ confidence intervals for the parameters of the mixture model (Supplementary Table 3) using the MclustBootstrap function ${ }^{43}$.

Heatmap. The heatmap was performed using XLSTAT (Addinsoft).

ATAC-seq and RNA-seq. All ATAC-seq datasets (from 13 NPC clones) used for this study were from Xu et al., 2017. Coordinates were lifted over from $\mathrm{mm} 9$ to mm10 using UCSC LiftOver. RNA-seq datasets from nine NPC clones used in this study were obtained from previously published work ${ }^{11,31}$. For the additional seven NPC clones and five astrocytes samples, total RNA $(1 \mu \mathrm{g})$ were prepared and used for polyA enrichment or ribosomal RNA depletion (RiboMinus Eukaryote System V2, Ambion), library preparation (TruSeq Stranded RNA-seq Library Prep, Illumina) and 75 or $100 \mathrm{bp}$ paired-end sequencing using a HiSeq2500. All biological replicates were merged. Strain-specific variants (CAST/EiJ and 129S1/SvImJ) were downloaded from the Sanger Mouse Genomes Project (ftp://ftp-mouse.sanger.ac.uk/ REL-1505-SNPs_Indels/mgp.v5.merged.snps_all.dbSNP142.vcf.gz). Strain-specific SNPs were used to generate a reference FASTA file N-masked at high-quality homozygous SNPs differing between the two parental strains with SNPsplit (0.3.2). Reads were aligned to the $\mathrm{N}$-masked genome using Tophat (2.1.0)/Bowtie2 (2-2.2.5) with the following parameters: --reorder -p 8 -D 70 -R 3 -N 0 -L 20 -i S, 1,0.50. Reads spanning the $\mathrm{N}$-masked sites were assigned to the maternal or paternal allele using SNPsplit (0.3.2). If both reads are assigned to the same parental allele or one read is assigned to one parental allele and the other is unassigned, they are classified as allele-specific. Reads without SNP information, with conflicting allele assignment, or unexpected alleles at polymorphic sites are flagged as unassigned. Read counts were assigned using allele-specific and total BAM files with FeatureCounts (1.5.1) with the following parameters: $-\mathrm{C}-\mathrm{p}-\mathrm{s} 2$.

Raw (FASTQ files) and processed data were deposited in GEO under accession number GSE148348. Details about all clones, names and references can be found in Supplementary Table 4.

Analysis of the RNA-seq in NPCs and astrocytes. Allelic expression ratios (Cast/ total allele-specific reads) were calculated for each gene, in each NPC clone and the corresponding astrocyte population. Values with less than 20 allele-specific reads were excluded. Among the 394 genes previously categorised as RME ${ }^{11}, 195$ could be assessed in at least one NPC-astrocyte pair.

Analysis of the association between ATAC-seq and RNA-seq allelic ratios. For each gene of interest, allelic accessibility and allelic expression ratios (Cast/total allele-specific reads) were calculated for each ATAC-seq and RNA-seq peak within $\pm 3 \mathrm{Mb}$ of the TSS of the gene considered. Peaks with less than ten allelespecific reads were excluded from the analysis. Only peaks with more than three values and at least two clones with an expression bias $(<33 \%$ or $>66 \%)$ were included in the regression. For each gene, linear regression was performed between the RNA-seq allelic expression ratio and the allelic accessibility ratio for each ATAC-seq peak.

DNA methylation analysis. Genomic DNA was extracted from NPC cell pellets using the DNeasy Blood and Tissue Kit (Qiagen) and bisulfite-converted using Epitect Bisulfite Kit (Qiagen). Bisulfite-treated DNA was amplified using primers for the CpG islands found at the promoters of the genes Acyp2 and Bag3. PCR products were analyzed by MALDI-TOF mass spectrometry after in vitro transcription and specific cleavage by Sequenom (Epityper analysis) ${ }^{11}$. Primer sequences can be found in Supplementary Table 8 .

Western blot analysis. NPC cell pellets were resuspended in $100 \mu \mathrm{L}$ RIPA buffer (Tris- $\mathrm{HCl} 50 \mathrm{mM} \mathrm{pH} \mathrm{8.0-8.5,} \mathrm{NaCl} 150 \mathrm{mM}$, Triton X-100 1\%, Sodium deoxycholate $0.5 \%$, SDS $0.1 \%$ ) with protease inhibitor (Roche), and sonicated using a Bioruptor (Diagenode, medium power, $3 \times 15 \mathrm{sec}$ ). Protein concentration was measured using the Bradford assay (Bio-Rad), and adjusted to $1 \mu \mathrm{g} / \mu \mathrm{L}$. LDS sample buffer (Invitrogen) and DTT $(250 \mathrm{mM})$ were added, then samples were heated at $95^{\circ} \mathrm{C}$ for $5 \mathrm{~min}$. About $10 \mu \mathrm{L}$ of each sample were loaded on a NuPAGE $12 \%$ BisTris gel, with a protein ladder (PageRuler 26616, Thermo Scientific), and electrophoresis was performed on an Invitrogen system, with a MOPS SDS buffer (NuPAGE, Invitrogen). The transfer to a nitrocellulose membrane was performed using a Bio-Rad system, in Tris $25 \mathrm{mM}$, Glycine $192 \mathrm{mM}$, methanol $20 \%$, for $1 \mathrm{~h}$ at $100 \mathrm{~V}$. The membrane was blocked for $1 \mathrm{~h}$ in TBS-T $0.1 \%$, BSA 5\%. Primary antibodies against BAG3 (rabbit polyclonal, 10599-1-AP, Proteintech) and GAPDH (mouse monoclonal, ab9484, Abcam) were incubated overnight at 1/ 1000e. Secondary antibodies anti-mouse conjugated with Alexa Fluor 546 (Invitrogen, A-11030) and anti-rabbit conjugated with Alexa Fluor 488 (Invitrogen, A11034 ) at $1 / 5000$ e were incubated for $1 \mathrm{~h}$ at room temperature. The membrane was then washed and imaged with a ChemiDoc MP Imaging System (Bio-Rad). The level of full-length BAG3 was normalised to the level of GAPDH for each clone analysed.

Epidrug screen. We used an 'epigenetics compound library' from Selleckchem, containing 181 compounds (Supplementary Table 5), dissolved in DMSO or water (172 and 9 compounds, respectively). NPCs were plated at a density of $7.5 \times 10^{4}$ cells $/ \mathrm{cm}^{2}$ in 96 -well plates coated with gelatin $0.1 \%$. Six hours later, compounds were added to each well at a final concentration of $10 \mu \mathrm{M}$ and $0.5 \%$ of solvent (DMSO or water). Cells were collected $48 \mathrm{~h}$ later for lysis, and reverse transcription was performed using the Cells-to-Ct kit (Applied Biosystems) directly in 96-well plates. The allelic expression ratio of Bag3 was then measured using pyrosequencing after PCR. The 11 best hits were then tested following the same protocol, in 24-well plates. The dose-response curves were performed in 6-well plates, with various concentrations of Dacinostat and Decitabine and a constant concentration of DMSO (0.5\%). The final validation was performed in a six-well plate format, using new batches of Dacinostat and Decitabine (Selleck Chemicals), dissolved in DMSO; cells were treated for $24 \mathrm{~h}$ with $10 \mu \mathrm{M}$ of drugs, in 0.5\% DMSO. For assays in 24-well and six-well plates, cells were harvested and RNAs extracted using the Qiagen RNeasy plus mini kit, as described earlier.

Mice. The experiments were conducted with 15-week-old female C57Bl/6 J mice (Charles River, France). The animals were housed in groups, in a 12-h light-dark cycle, in stable conditions of temperature, with food and water ad libitum. Experiments were in accordance with the European Community Council Directive 2010/63/EU and approved by the ethics committee of the Institut Curie CEEAIC\#118 and authorised by the Ministère de l'Education Nationale, de l'Enseignement Supérieur et de la Recherche (APAFIS\#8812-2017020611033784 v2). 
RNA-FISH. RNA-FISH probes were prepared by Nick Translation (Abbott) following the manufacturer's instructions and with a $4 \mathrm{~h}$ enzymatic incubation, using Atto-488 conjugated dUTP (Jena Bioscience), and the BAC clones RP23-399D1 and RP24-310A20 (Chori BACPAC Resources Center) for the App and the Grik2 genes, respectively.

Mice were quickly and deeply anaesthetised with pentobarbital (500 mg/kg, i.p.) and transcardially perfused with $40 \mathrm{~g} / \mathrm{L}$ formaldehyde in phosphate-buffered saline (PBS) for $5 \mathrm{~min}$. Brains were dissected and kept at $4{ }^{\circ} \mathrm{C}$ for $12 \mathrm{~h}$ in a PBS solution containing $15 \%$ sucrose and $2 \mathrm{mM}$ VRC (vanadium ribonucleoside complex) before being flash frozen in isopentane $\left(1 \mathrm{~min}\right.$ at $\left.-30^{\circ} \mathrm{C}\right)$ and stored at $-80^{\circ} \mathrm{C}$. Serial coronal sections ( $30 \mu \mathrm{m}$ thick) were made using a cryostat (Leica), mounted onto Superfrost Plus slides, and stored at $-80^{\circ} \mathrm{C}$.

Sections were fixed for $5 \mathrm{~min}$ in $30 \mathrm{~g} / \mathrm{L}$ formaldehyde in PBS, and washed with PBS, $2 \mathrm{mM}$ VRC, at $4{ }^{\circ} \mathrm{C}$. They were permeabilized in $0.5 \%$ Triton X-100, PBS $1 \mathrm{X}$, $2 \mathrm{mM}$ VRC for $5 \mathrm{~min}$ before being washed with $70 \%$ ethanol. To remove autofluorescence, sections were incubated in $0.1 \%$ (w/v) Sudan Black B, 70\% ethanol, for $10 \mathrm{~min}$ at RT, then quickly rinsed in $70 \%$ ethanol. Sections were equilibrated in $50 \%(\mathrm{v} / \mathrm{v})$ formamide, $1 \mathrm{X}$ SSC $(150 \mathrm{mM} \mathrm{NaCl}, 15 \mathrm{mM}$ sodium

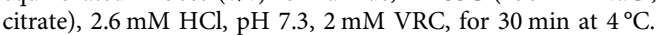

About $40 \mathrm{ng}$ of labelled DNA probe was precipitated together with $3 \mu \mathrm{g}$ Cot-1 DNA and $10 \mu \mathrm{g}$ salmon sperm DNA, before being resuspended in $8 \mu \mathrm{L}$ formamide, denatured at $75^{\circ} \mathrm{C}$, then incubated at $37^{\circ} \mathrm{C}$ for $30 \mathrm{~min}$ for Cot-1 competition. The labeled DNA probe was then hybridised in the presence of Cot-1 and salmon sperm DNA, for $16-24 \mathrm{~h}$ at $37^{\circ} \mathrm{C}$ in $16 \mu \mathrm{L}$ of the following solution: $50 \%(\mathrm{v} / \mathrm{v})$ formamide, 1X SSC, $10 \mathrm{mM}$ VRC, $10 \%(\mathrm{w} / \mathrm{v})$ dextran sulfate, $0.2 \%(\mathrm{w} / \mathrm{v}) \mathrm{BSA}$. After hybridisation, slides were washed two times in $50 \%$ formamide/ $2 \mathrm{X}$ SSC at $42^{\circ} \mathrm{C}$ for $30 \mathrm{~min}$, then washed and counterstained with $10 \mathrm{nM}$ TO-PRO-3 in $2 \mathrm{X}$ SSC at $42^{\circ} \mathrm{C}$ for $2 \times 30 \mathrm{~min}$. The slides were mounted in Vectashield (Vector Laboratories, USA).

Microscopy. A laser scanning spinning disk confocal microscope (Roper/Nikon) with a $40 \times$ objective was used for image acquisition, which was carried out at the Cell and Tissue Imaging Platform (PICT-IBiSA) at Institut Curie.

Statistical analysis for RNA-FISH. We tested whether the monoallelism measured by RNA-FISH could be explained by transcriptional bursts and/or by the sensitivity of detection. We considered the probability $\left(P_{\text {detect }}\right)$ of detecting the nascent RNA for an active allele to be given by:

$P_{\text {detect }}=P_{\mathrm{ON}} \times P_{\text {sense, }}$, where $P_{\mathrm{ON}}$ is the probability to be in the ON phase of transcription, and $P_{\text {sense }}$ is the sensitivity of the RNA-FISH, $\left(P_{\text {sense }}\right.$ depends on the detection power of RNA-FISH and on the expression level).

For a biallelic gene with identical and unsynchronised alleles, we estimated $P_{\text {detect }}$ by:

$P_{\text {detect }}=n_{\text {tot }} /\left(2 \times N_{\text {cells }}\right)$, where $n_{\text {tot }}$ is the total number of pinpoints detected over $N$ cells, each comprising two alleles.

We reasoned that, if the observed monoallelism was due to transcriptional bursts or to lack of detection, the probabilities of detecting each allele should be independent and identical, in which case, the number of alleles observed would follow a binomial distribution $\mathrm{B}\left(2, P_{\text {detect }}\right)$. We tested this hypothesis using a $\chi^{2}$ goodness of fit test with one degree of freedom.

Reporting summary. Further information on research design is available in the Nature Research Reporting Summary linked to this article.

\section{Data availability}

The data that support this study are available from the corresponding authors upon reasonable request. The raw RNA sequencing data (FASTQ files) generated in this study have been deposited in the GEO database under accession code GSE148348. Previously published sequencing data used in this study are available in the GEO database under accession codes GSE54016, GSE72697 and GSE84646. Source data are provided with this paper.

Received: 22 May 2020; Accepted: 19 August 2021;

Published online: 09 September 2021

\section{References}

1. Joho, R. \& Weissman, I. L. V-J joining of immunoglobulin kappa genes only occurs on one homologous chromosome. Nature 284, 179-181 (1980).

2. Lawson, H. A., Cheverud, J. M. \& Wolf, J. B. Genomic imprinting and parentof-origin effects on complex traits. Nat. Rev. Genet. 14, 609-617 (2013).

3. Galupa, R. \& Heard, E. X-chromosome inactivation: new insights into cis and trans regulation. Curr. Opin. Genet. Dev. 31, 57-66 (2015).

4. Monahan, K. \& Lomvardas, S. Monoallelic expression of olfactory receptors. Annu. Rev. Cell Dev. Biol. 31, 721-740 (2015).
5. Gendrel, A. V., Marion-Poll, L., Katoh, K. \& Heard, E. Random monoallelic expression of genes on autosomes: parallels with X-chromosome inactivation. Semin. Cell Dev. Biol. 56, 100-110 (2016)

6. Gimelbrant, A., Hutchinson, J. N., Thompson, B. R. \& Chess, A. Widespread monoallelic expression on human autosomes. Science $\mathbf{3 1 8}$, 1136-1140 (2007).

7. Zwemer, L. M. et al. Autosomal monoallelic expression in the mouse. Genome Biol. 13, R10 (2012).

8. Jeffries, A. R. et al. Stochastic choice of allelic expression in human neural stem cells. Stem Cells 30, 1938-1947 (2012).

9. Li, S. M. et al. Transcriptome-wide survey of mouse CNS-derived cells reveals monoallelic expression within novel gene families. PLoS ONE 7, e31751 (2012).

10. Eckersley-Maslin, M. A. et al. Random monoallelic gene expression increases upon embryonic stem cell differentiation. Dev. Cell 28, 351-365 (2014).

11. Gendrel, A. V. et al. Developmental dynamics and disease potential of random monoallelic gene expression. Dev. Cell 28, 366-380 (2014).

12. Reinius, B. et al. Analysis of allelic expression patterns in clonal somatic cells by single-cell RNA-seq. Nat. Genet. 48, 1430-1435 (2016).

13. $\mathrm{Xu}$, J. et al. Landscape of monoallelic DNA accessibility in mouse embryonic stem cells and neural progenitor cells. Nat. Genet. 49, 377-386 (2017)

14. Branciamore, S. et al. Frequent monoallelic or skewed expression for developmental genes in CNS-derived cells and evidence for balancing selection. Proc. Natl Acad. Sci. USA 115, E10379-E10386 (2018).

15. Savova, V., Patsenker, J., Vigneau, S. \& Gimelbrant, A. A. dbMAE: the database of autosomal monoallelic expression. Nucleic Acids Res. 44 D753-D756 (2016).

16. Gregg, $\mathrm{C}$. The emerging landscape of in vitro and in vivo epigenetic allelic effects. F1000Research 6, 2108 (2017).

17. Vigneau, S., Vinogradova, S., Savova, V. \& Gimelbrant, A. High prevalence of clonal monoallelic expression. Nat. Genet. 50, 1198-1199 (2018).

18. Reinius, B. \& Sandberg, R. Reply to 'high prevalence of clonal monoallelic expression'. Nat. Genet. 50, 1199-1200 (2018).

19. Huang, W. C. et al. Diverse non-genetic, allele-specific expression effects shape genetic architecture at the cellular level in the mammalian brain. Neuron 93, 1094-1109 (2017).

20. Reinius, B. \& Sandberg, R. Random monoallelic expression of autosomal genes: stochastic transcription and allele-level regulation. Nat. Rev. Genet. 16, 653-664 (2015).

21. Conti, L. et al. Niche-independent symmetrical self-renewal of a mammalian tissue stem cell. PLoS Biol. 3, e283 (2005).

22. Wang, Y. et al. Association between ACYP2 polymorphisms and the risk of renal cell cancer. Mol. Genet. Genom. Med. 7, e966 (2019).

23. Blacker, D. et al. Alpha-2 macroglobulin is genetically associated with Alzheimer disease. Nat. Genet. 19, 357-360 (1998).

24. Homma, S. et al. BAG3 deficiency results in fulminant myopathy and early lethality. Am. J. Pathol. 169, 761-773 (2006).

25. Wayne, S. et al. Mutations in the transcriptional activator EYA4 cause lateonset deafness at the DFNA10 locus. Hum. Mol. Genet. 10, 195-200 (2001).

26. Cordoba, M., Rodriguez, S., Gonzalez Moron, D., Medina, N. \& Kauffman, M. A. Expanding the spectrum of Grik2 mutations: intellectual disability, behavioural disorder, epilepsy and dystonia. Clin. Genet. 87, 293-295 (2015).

27. Zhang, J. et al. Overexpression of BAG3 attenuates hypoxia-induced cardiomyocyte apoptosis by inducing autophagy. Cell. Physiol. Biochem. 39, 491-500 (2016)

28. Li, J. et al. Down-regulation of BAG3 inhibits proliferation and promotes apoptosis of glioblastoma multiforme through BAG3/HSP70/HIF-1alpha signaling pathway. Int. J. Clin. Exp. Pathol. 11, 4305-4318 (2018).

29. Bureau, I., Bischoff, S., Heinemann, S. F. \& Mulle, C. Kainate receptormediated responses in the CA1 field of wild-type and GluR6-deficient mice. $J$. Neurosci. 19, 653-663 (1999).

30. Telfeian, A. E., Federoff, H. J., Leone, P., During, M. J. \& Williamson, A. Overexpression of GluR6 in rat hippocampus produces seizures and spontaneous nonsynaptic bursting in vitro. Neurobiol. Dis. 7, 362-374 (2000).

31. Giorgetti, L. et al. Structural organization of the inactive X chromosome in the mouse. Nature 535, 575-579 (2016).

32. Nica, A. C. \& Dermitzakis, E. T. Expression quantitative trait loci: present and future. Philos. Trans. R. Soc. Lond. B Biol. Sci. 368, 20120362 (2013).

33. Zou, J. et al. Leveraging allelic imbalance to refine fine-mapping for eQTL studies. PLoS Genet. 15, e1008481 (2019).

34. Aseem, O., Barth, J. L., Klatt, S. C., Smith, B. T. \& Argraves, W. S. Cubilin expression is monoallelic and epigenetically augmented via PPARs. BMC Genomics 14, 405 (2013).

35. Ng, K. K. et al. A stochastic epigenetic switch controls the dynamics of T-cell lineage commitment. eLife https://doi.org/10.7554/eLife.37851 (2018).

36. Khamlichi, A. A. \& Feil, R. Parallels between mammalian mechanisms of monoallelic gene expression. Trends Genet. 34, 954-971 (2018). 
37. Nag, A. et al. Chromatin signature of widespread monoallelic expression. eLife 2, e01256 (2013)

38. Savol, A. J. et al. Genome-wide identification of autosomal genes with allelic imbalance of chromatin state. PLoS ONE 12, e0182568 (2017).

39. Norton, N. et al. Genome-wide studies of copy number variation and exome sequencing identify rare variants in BAG3 as a cause of dilated cardiomyopathy. Am. J. Hum. Genet. 88, 273-282 (2011).

40. Myers, V. D. et al. Haplo-insufficiency of Bcl2-associated athanogene 3 in mice results in progressive left ventricular dysfunction, beta-adrenergic insensitivity, and increased apoptosis. J. Cell. Physiol. 233, 6319-6326 (2018).

41. Scrucca, L., Fop, M., Murphy, T. B. \& Raftery, A. E. mclust 5: clustering, classification and density estimation using Gaussian finite mixture models. $R$. J. 8, 289-317 (2016).

42. McLachlan, G. J. \& Rathnayake, S. On the number of components in a Gaussian mixture model. Wires Data Min. Knowl. 4, 341-355 (2014).

43. O'Hagan, A., Murphy, T. B., Scrucca, L. \& Gormley, I. C. Investigation of parameter uncertainty in clustering using a Gaussian mixture model via jackknife, bootstrap and weighted likelihood bootstrap. Comput. Stat. 34, 1779-1813 (2019).

\section{Acknowledgements}

We thank Isabelle Grandjean, Colin Jouhanneau and Cédrick Pauchard (In vivo experiments platform of Institut Curie) for the animal care, Aude Muzerelle for histology advice, Agnès Le Saux and Patricia Diabangouaya for technical assistance, and members of the Heard lab for stimulating discussions. We are grateful to Joost Gribnau (Oncode Institute, Utrecht, NL) for initially providing the F1-21.6 and F1-23 ESC lines. Microscopy and image analysis were carried out at the Cell and Tissue Imaging Platform of the Genetics and Developmental Biology department (UMR3215/U934) of Institut Curie, member of France-Bioimaging (ANR-10-INSB-04). High-throughput sequencing was performed by the ICGex NGS platform of the Institut Curie platform and the Stanford Functional Genomics Facility. The ICGEx NGS platform is supported by grants ANR-10EQPX-03 (Equipex) and ANR-10-INBS-09-08 (France Génomique Consortium) from the ANR ('Investissements d'Avenir' programme), the Canceropole Ile-de-France and the SiRIC-Curie programme (SiRIC grant INCa-DGOS-4654). This work was supported by Biogen, an ERC Advanced Investigator award ERC-ADG-2014 671027 and by grants from the Fondation Vaincre Alzheimer (FR-17068) and France Parkinson (FP 2017) to E.H., and by NIH RM1-HG007735 (to H.Y.C.). H.Y.C. is an Investigator of the Howard Hughes Medical Institute. L.M.-P. was funded by a fellowship from the Fondation pour la Recherche Médicale (FRM, SPF20151234950).

\section{Author contributions}

A.-V.G., E.H. and L.M.-P. conceptualised the study, designed the experiments and co-supervised this work. A.-V.G., B.F., L.M.-P. and M.A. performed in vitro experiments. L.M.-P. carried out RNA-FISH experiments. B.F. performed the qPCR and pyrosequencing experiments. A.C.C., A.-V.G., H.Y.C. and M.A. generated RNA-seq data. D.Z., F.M., L.S. and L.M.-P. analyzed data. A.-V.G., E.H. and L.M.-P. interpreted and discussed the data. A.-V.G., E.H. and L.M.-P. wrote the manuscript, with critical revision of the article by A.C.C., D.Z. and H.Y.C. All the authors approved the final version of the manuscript.

\section{Funding}

Open Access funding enabled and organized by Projekt DEAL.

\section{Competing interests}

H.Y.C. is a co-founder of Accent Therapeutics, Boundless Bio, and an advisor to 10x Genomics, Arsenal Biosciences, and Spring Discovery. The remaining authors declare no competing interests.

\section{Additional information}

Supplementary information The online version contains supplementary material available at https://doi.org/10.1038/s41467-021-25630-3.

Correspondence and requests for materials should be addressed to L.M-P., A.-V.G. or E.H.

Peer review information Nature Communications thanks John Goutsias and the anonymous reviewer(s) for their contribution to the peer review of this work.

Reprints and permission information is available at http://www.nature.com/reprints

Publisher's note Springer Nature remains neutral with regard to jurisdictional claims in published maps and institutional affiliations.

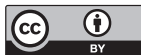

Open Access This article is licensed under a Creative Commons Attribution 4.0 International License, which permits use, sharing, adaptation, distribution and reproduction in any medium or format, as long as you give appropriate credit to the original author(s) and the source, provide a link to the Creative Commons license, and indicate if changes were made. The images or other third party material in this article are included in the article's Creative Commons license, unless indicated otherwise in a credit line to the material. If material is not included in the article's Creative Commons license and your intended use is not permitted by statutory regulation or exceeds the permitted use, you will need to obtain permission directly from the copyright holder. To view a copy of this license, visit http://creativecommons.org/ licenses/by/4.0/.

(c) The Author(s) 2021, corrected publication 2021 